CERN-TH-2016-192

\title{
Weakening Gravity on Redshift-Survey Scales with Kinetic Matter Mixing
}

\author{
Guido D'Amico ${ }^{\mathrm{a}}$, Zhiqi Huang ${ }^{\mathrm{b}}$, \\ Michele Mancarella ${ }^{\mathrm{c}, \mathrm{d}}$ and Filippo Vernizzi ${ }^{\mathrm{c}, \mathrm{d}}$ \\ ${ }^{a}$ Theoretical Physics Department, CERN, Geneva, Switzerland \\ b School of Physics and Astronomy, Sun Yat-Sen University, \\ 135 Xingang Xi Road, 510275, Guangzhou, China \\ c CEA, IPhT, 91191 Gif-sur-Yvette cédex, France \\ CNRS, URA-2306, 91191 Gif-sur-Yvette cédex, France \\ d Université Paris Sud, 15 rue George Clémenceau, 91405, Orsay, France
}

September 6, 2016

\begin{abstract}
We explore general scalar-tensor models in the presence of a kinetic mixing between matter and the scalar field, which we call Kinetic Matter Mixing. In the frame where gravity is demixed from the scalar this is due to disformal couplings of matter species to the gravitational sector, with disformal coefficients that depend on the gradient of the scalar field. In the frame where matter is minimally coupled, it originates from the so-called beyond Horndeski quadratic Lagrangian. We extend the Effective Theory of Interacting Dark Energy by allowing disformal coupling coefficients to depend on the gradient of the scalar field as well. In this very general approach, we derive the conditions to avoid ghost and gradient instabilities and we define Kinetic Matter Mixing independently of the frame metric used to described the action. We study its phenomenological consequences for a $\Lambda$ CDM background evolution, first analytically on small scales. Then, we compute the matter power spectrum and the angular spectra of the CMB anisotropies and the CMB lensing potential, on all scales. We employ the public version of COOP, a numerical Einstein-Boltzmann solver that implements very general scalar-tensor modifications of gravity. Rather uniquely, Kinetic Matter Mixing weakens gravity on short scales, predicting a lower $\sigma_{8}$ with respect to the $\Lambda \mathrm{CDM}$ case. We propose this as a possible solution to the tension between the CMB best-fit model and low-redshift observables.
\end{abstract}




\section{Contents}

1 Introduction $\quad 2$

2 Effective Theory of Dark Energy with Kinetic Matter Mixing 5

2.1 Gravitational and matter actions . . . . . . . . . . . . . . . 5

2.2 Matter couplings and stability conditions . . . . . . . . . . . . . . 7

$2.3(\partial \phi)^{2}$-dependent disformal transformations $\ldots \ldots \ldots \ldots$

3 Short-scale dynamics $\quad 10$

3.1 Oscillatory regime and normal modes . . . . . . . . . . . . . . . . 10

3.2 Quasi-static regime . . . . . . . . . . . . . . . . . . . 12

4 Observational signatures of Kinetic Matter Mixing $\quad 14$

4.1 Matter power spectrum . . . . . . . . . . . . . . . . . 15

4.2 Cosmic Microwave Background . . . . . . . . . . . . . . . . . . . 18

4.3 Short-scale tension . . . . . . . . . . . . . . . . . . . 20

5 Summary and conclusions $\quad 23$

A Quadratic action and stability for multiple species $\quad 24$

$\begin{array}{ll}\text { B Quadratic action in Newtonian gauge } & 27\end{array}$

$\begin{array}{lr}\text { C From the oscillating to the quasi-static regime } & 28\end{array}$

D Observational signatures of Kinetic Braiding 29

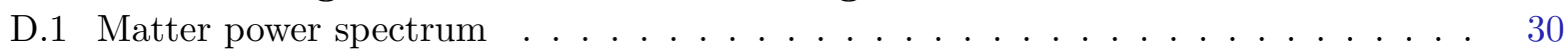

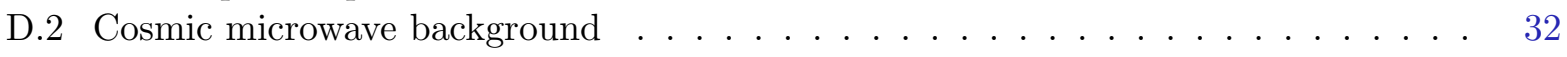

\section{Introduction}

A key goal of current and future cosmic surveys is to constrain or possibly detect deviations from the standard $\Lambda \mathrm{CDM}$ model, which are expected if the origin of the present accelerated expansion is not a cosmological constant, but a dynamical field or a modification of General Relativity (see e.g. $[1,2]$ ). To deal with the fact that there are many dark energy and modified gravity models (see for instance [3,4]), effective approaches that describe these deviations for a large number of models in terms of a few time-dependent parameters have been proposed in the literature [5-16]. In most cases, these approaches are limited to a description of cosmological perturbations around a Friedmann-Lemaittre-Robertson-Walker (FLRW) background in the linear regime (see however $[17,18]$ for some nonlinear aspects), applicable to scales above $\sim 10 \mathrm{Mpc}$, where deviations from General Relativity are not yet well tested.

This work focuses on the so-called Effective Theory of Dark Energy. Formulated for single scalar field models - i.e. models where the time diffeomorphisms are broken while leaving the spatial ones preserved - in this approach the unitary (or uniform field) gauge action is given as the sum of all possible geometrical elements constructed from the metric and its derivatives that are invariant 
under the preserved diffs, i.e. the spatial ones $[19,20]$. It has been derived and studied for minimally and nonminimally coupled dark energy models, respectively, in [5] and [6,8] (see [10,21,22] for reviews). When restricting to the lowest order in derivatives, the final second-order action contains five free functions of time that parametrize any deviation from $\Lambda$ CDM. As shown in [8], four of these functions describe cosmological perturbations of effective theories of dark energy or modified gravity within the Horndeski class, i.e. those with quadratic gravitational action with the same structure as Horndeski theories [23-25]. This description has been reformulated in [26] in terms of dimensionless functions that clearly parametrize deviations from General Relativity. The fifth function, denoted as $\alpha_{\mathrm{H}}$, describes scalar field models extending the Horndeski class, such as, e.g., the theories "beyond Horndeski" proposed in [27,28] (see [29] for an earlier proposal of theories beyond Horndeski). The Effective Theory formulation has been used to explore the observational consequences of deviations from $\Lambda \mathrm{CDM}$ (see for instance [30-41]). In this direction, a few EinsteinBoltzmann solvers have been recently developed and employed [42-47].

References $[6,8]$ assumed that all matter species are minimally coupled to the same metric, which we call Jordan frame metric for convenience. In general, however, there is no reason to impose this restriction. The universality of couplings is very well tested on Solar System scales [48] but on cosmological scales constraints are much weaker and different species could have distinct couplings to the gravitational sector. If matter is universally but nonminimally coupled to the gravitational sector, in most cases it is convenient to perform a field redefinition of the metric that brings the system into the Jordan frame, where matter is minimally coupled. In general, this frame transformation depends on the scalar field and its derivatives and, as long as it is regular and invertible, it cannot change the physics (see e.g. [49]). The advantage of using the Jordan frame to derive predictions is that only the gravitational sector is non-standard; thus, one does not need to care about modifications of non-gravitational forces, which would otherwise greatly complicate the analysis.

Along this line of thought, recently Ref. [50] extended the effective approach of [6,8] to allow for distinct conformal and disformal couplings of matter species to the gravitational sector. The treatment was restricted to effective theories within the Horndeski class and to conformal and disformal factors that depend only on the scalar field (not on its gradients). In this case, the full quadratic action depends on the four functions describing the gravitational sector and on two extra functions per species, describing the coupling to the scalar. However, two of these functions are redundant, because the structure of the action is preserved under transformations of the reference metric. This is expected, as it was shown that the structure of the Horndeski Lagrangians is preserved under disformal transformations with both conformal and disformal coefficients independent of the scalar field gradient [51].

The phenomenological aspects of general modifications of gravity described by Ref. [50] was studied in Ref. [35], where constraints on the effective descriptions were derived from three observables: the galaxy and weak-lensing power spectra and the correlation between the Integrated Sachs-Wolfe (ISW) effect and the galaxy distribution. However, the study was restricted to the quasi-static limit, which is reliable on short enough scales and at late times, once the oscillations of the scalar fluctuations have been damped by the expansion of the universe. While this approximation is fairly good for current and future galaxy and weak lensing surveys, ${ }^{1}$ it fails on large scales or high redshifts.

\footnotetext{
${ }^{1}$ The quasi-static approximation typically fails on scales $k \lesssim a H / c_{s}$, where $c_{s}$ is the sound speed of fluctuations of the scalar. As shown in [52], this approximation should be reliable for surveys such as Euclid as long as the sound speed exceeds $10 \%$ of the speed of light, i.e. $c_{s} \gtrsim 0.1$.
} 
In this article we go one step forward, in two directions. First, in Sec. 2 we extend the treatment of Ref. [50] and include in the gravitational action the fifth time-dependent function, $\alpha_{\mathrm{H}}$, describing models extending the Horndeski class. As shown in [27,28], the structure of the Lagrangian of theories beyond Horndeski is preserved under a disformal transformation of the metric with disformal coefficient that depends as well on the gradient of the scalar field, i.e. of the form

$$
\tilde{g}_{\mu \nu}=C(\phi) g_{\mu \nu}+D(\phi, X) \partial_{\mu} \phi \partial_{\nu} \phi, \quad X \equiv g^{\mu \nu} \partial_{\mu} \phi \partial_{\nu} \phi .
$$

Thus, in the following we consider the possibility that matter couples to a Jordan frame metric of this form. ${ }^{2}$ In particular, we denote the conformal and disformal coefficients of the nonminimal coupling of matter respectively as $C_{\mathrm{m}}(\phi)$ and $D_{\mathrm{m}}(\phi, X)$ (which can be distinct for different species).

As shown in Sec. 2, the dependence of the disformal coupling on the derivative of the field introduces a kinetic mixing between the scalar and matter, which hereafter we call Kinetic Matter Mixing (KMM), that has rather unique observational effects, as discussed below. To parametrize this direct kinetic coupling we introduce an additional function of time,

$$
\alpha_{\mathrm{X}, \mathrm{m}}=\frac{X^{2}}{C_{\mathrm{m}}} \frac{\partial D_{\mathrm{m}}}{\partial X}
$$

where the right-hand side is evaluated on the background. Thus, the full quadratic action depends now on five functions describing the gravitational sector and three functions per species, describing the matter couplings. The structure of this action is preserved under transformations of the reference metric of the form (1.1). Remarkably, $\alpha_{\mathrm{X}, \mathrm{m}}$ is transformed into the beyond Horndeski parameter $\alpha_{\mathrm{H}}$ under a transformation which sets to zero the disformal coupling. Since KMM is a truely physical effect, it is possible to define a combination of these two parameters, proportional to $\left(\alpha_{\mathrm{H}}-\alpha_{\mathrm{X}, \mathrm{m}}\right)^{2}$ (c.f. eq. (2.17) below), that encodes in a frame-independent way the degree of kinetic mixing between matter and the scalar.

While in Sec. 2 we assume for simplicity that matter couples universally to the same Jordan frame metric, in App. A we extend this treatment to multiple species with distinct couplings. Taking into account the invariance under the disformal transformation (1.1), which reduces the number of independent functions of time by three, the whole system depends on a total of $2+3 N_{S}$ independent functions of time, where $N_{S}$ is the number of matter species.

In the rest of the paper we assume that matter is universally coupled to the gravitational sector and work in the Jordan frame, where the coupling is minimal. In this frame, KMM is encoded in the beyond Horndeski parameter $\alpha_{\mathrm{H}}$. We then extend the treatment of Ref. [35] and explore the phenomenological consequences of general late-time modifications of gravity including beyond Horndeski theories (see also [58,59] and [60-63] for an earlier study of the observational consequences of beyond Horndeski theories, respectively in cosmology and astrophysics). In Sec. 3, we focus on short scales. In particular, we derive the eigenmodes of propagation of the scalar field and matter, which in the presence of a nonvanishing $\alpha_{\mathrm{H}}$ are mixed by their kinetic coupling. Moreover, we obtain the evolution equations in the quasi-static regime, which govern the dynamics once the oscillating modes have been damped by the expansion. Appendix B contains the full action of perturbations in Newtonian gauge, derived for completeness, while the transition between the oscillating regime and the quasi-static limit is discussed in App. C.

\footnotetext{
${ }^{2}$ Disformal transformations with $C=C(\phi, X)$ have been studied in the context of beyond Horndeski theories in $[29,53]$ and in the context of degenerate higher-order theories in [54-57].
} 
In Sec. 4 we go beyond the quasi-static approximation and explore the full range of cosmological scales using the linear Einstein-Boltzmann solver of Cosmology Object Oriented Package (COOP) [47], ${ }^{3}$ which solves cosmological perturbations including very general deviations from $\Lambda \mathrm{CDM}$ in terms of the Effective Theory of Dark Energy description [10]. In particular, assuming the background expansion history of $\Lambda \mathrm{CDM}$, we compute the matter power spectrum, the Cosmic Microwave Background (CMB) anisotropies angular power spectrum, and the CMB lensing potential angular spectrum in the presence of KMM, for a non vanishing $\alpha_{\mathrm{H}}$ parameter. As we will see, on "short" scales, i.e. for $k \gtrsim 10^{-3} h \mathrm{Mpc}^{-1}$, the quasi-static approximation provides the correct amplitude for the linear growth factor, which is scale independent and suppressed with respect to the $\Lambda \mathrm{CDM}$ case. On larger scales, we compute the linear matter growth analytically using a perturbative expansion in $\alpha_{\mathrm{H}}$ that confirms the numerical results. To contrast with the effects of $\alpha_{\mathrm{H}}$, in App. D we compute the same observables in the case of a kinetic mixing between the scalar field and gravity, the so called kinetic braiding [64,65] (see [5, 19] for an earlier study), and we find agreement with the results of Ref. [45]. We compare these results with the quasi-static approximation and a perturbative expansion in the braiding parameter. In contrast to kinetic braiding or other modifications of gravity within the Horndeski class, the exchange of fifth force in KMM suppresses the power of matter perturbations on redshift-survey scales. In Sec. 4.3, we study the possibility that the lack of power measured in the large scale structures and in tension with that inferred from the CMB anisotropies observed by Planck [66,67] can be explained by the KMM special signature. Finally, we conclude in Sec. 5.

\section{Effective Theory of Dark Energy with Kinetic Matter Mixing}

In this section we extend the treatment of [50], limited to Horndeski theories, and develop the unifying framework for dark energy and modified gravity that allows distinct conformal-disformal couplings of matter species to the gravitational sector, including beyond Horndeski theories. We show that the quadratic beyond Horndeski operator arises when transforming to the Jordan frame a disformal coupling of matter species which depends on the kinetic energy of the scalar field. In this setup, we derive the conditions to avoid ghost and gradient instabilities and discuss the disformal/conformal transformations of the gravitational and matter action. The reader only interested in the phenomenological aspects of KMM is invited to skip this section and go directly to Sec. 3, not before having retained eq. (2.5) as the second order action describing the gravitational sector.

\subsection{Gravitational and matter actions}

In the present work, following $[6,8]$ we assume that the gravitational sector is described by a four-dimensional metric $g_{\mu \nu}$ and a scalar field $\phi$. As usual, we choose a coordinate system such that the constant time hypersurfaces coincide with the uniform scalar field hypersurfaces. In this gauge, referred to as unitary gauge, the metric can be written in the ADM form,

$$
d s^{2}=-N^{2} d t^{2}+h_{i j}\left(d x^{i}+N^{i} d t\right)\left(d x^{j}+N^{j} d t\right)
$$

where $N$ is the lapse and $N^{i}$ the shift. In the following, a dot stands for a time derivative with respect to $t$, and $D_{i}$ denotes the covariant derivative associated with the three-dimensional spatial

\footnotetext{
${ }^{3}$ See http://www.cita.utoronto.ca/ zqhuang/ for documentation.
} 
metric $h_{i j}$. Spatial indices are lowered and raised with the spatial metric $h_{i j}$ or its inverse $h^{i j}$, respectively.

In the unitary gauge, a generic gravitational action can be written in terms of geometric quantities that are invariant under spatial diffeomorphisms [19,20]. Expressed in the ADM coordinates introduced above, these geometric quantities are the lapse $N$, the extrinsic curvature of the constant time hypersurfaces $K_{i j}$, whose components are given by

$$
K_{i j}=\frac{1}{2 N}\left(\dot{h}_{i j}-D_{i} N_{j}-D_{j} N_{i}\right),
$$

as well as the $3 \mathrm{~d}$ Ricci tensor of the constant time hypersurfaces $R_{i j}$ and, possibly, spatial derivatives of all these quantities. Thus, the gravitational action is generically of the form

$$
S_{\mathrm{g}}=\int d^{4} x \sqrt{-g} L\left(N, K_{i j}, R_{i j}, h_{i j}, D_{i} ; t\right) .
$$

To study linear perturbations, one needs to expand the action at second order around a homogeneous background. For the background geometry, we assume a spatially flat FLRW metric, $d s^{2}=-d t^{2}+a^{2}(t) d \vec{x}^{2}$. Its dynamics is governed by the background evolution equations and we refer the reader to Refs. $[8,10,50]$ for details on their derivation. We can now expand the gravitational action up to second order in perturbations. Fixing the background gauge $\bar{N}=1$, these are

$$
\delta N=N-1, \quad \delta K_{i j}=K_{i j}-H h_{i j},
$$

as well as $R_{i j}$, which is already a perturbation since its background value vanishes. It is convenient to introduce the time-dependent parameters $\alpha_{\mathrm{K}}, \alpha_{\mathrm{B}}, \alpha_{\mathrm{T}}$ [26] and $\alpha_{\mathrm{H}}$ [28] in terms of which the second-order gravitational action reads

$$
\begin{aligned}
& S_{\mathrm{g}}^{(2)}=\int d^{3} x d t a^{3} \frac{M^{2}}{2}\left[\delta K_{j}^{i} \delta K_{i}^{j}-\delta K^{2}+\left(1+\alpha_{\mathrm{H}}\right) R \delta N+\left(1+\alpha_{\mathrm{T}}\right) \delta_{2}\left(\sqrt{h} R / a^{3}\right)\right. \\
& \left.+\alpha_{\mathrm{K}} H^{2} \delta N^{2}+4 \alpha_{\mathrm{B}} H \delta K \delta N\right]
\end{aligned}
$$

where $\delta_{2}$ denotes taking the expansion at second order in perturbations. Another useful parameter is the variation of the effective Planck mass squared $M^{2}$,

$$
\alpha_{\mathrm{M}} \equiv \frac{d \ln M^{2}}{d \ln a}
$$

For the details on the derivation of the above action and the explicit definitions of the parameters $\alpha_{\mathrm{K}}, \alpha_{\mathrm{B}}, \alpha_{\mathrm{M}}, \alpha_{\mathrm{T}}$ and $\alpha_{\mathrm{H}}$ in terms of first and second derivatives of $L$ with respect to its arguments, we refer again the reader to Refs. $[8,10,50]$.

The gravitational action must be supplemented by a matter action $S_{\mathrm{m}}$,

$$
S_{\mathrm{m}}=\int d^{4} x \sqrt{-\check{g}} L_{I}\left(\check{g}_{\mu \nu}, \psi\right),
$$

where $\check{g}_{\mu \nu}$ is the Jordan-frame metric. In order to describe dark energy and modified gravity scenarios where the scalar and matter can be kinetically mixed, we assume that this metric is conformally and disformally related to the gravitational metric $g_{\mu \nu}$ by

$$
\check{g}_{\mu \nu}=C_{\mathrm{m}}^{(\phi)}(\phi) g_{\mu \nu}+D_{\mathrm{m}}^{(\phi)}(\phi, X) \partial_{\mu} \phi \partial_{\nu} \phi
$$


Contrarily to the disformal coupling presented in [50], here $D_{\mathrm{m}}^{(\phi)}$ can also depend on $X$, to allow for a kinetic mixing. In Appendix $\mathrm{A}$ we generalize to the case where matter is made of several species, each of which is coupled to a different metric.

To conclude, we notice that the variation of the matter action $S_{\mathrm{m}}$ with respect to the metric $g_{\mu \nu}$ defines the energy-momentum tensor, according to the standard expression

$$
T^{\mu \nu} \equiv \frac{2}{\sqrt{-g}} \frac{\delta S_{\mathrm{m}}}{\delta g_{\mu \nu}}
$$

This definition applies even if matter is minimally coupled to a metric $\check{g}_{\mu \nu}$ that differs from $g_{\mu \nu}$. In the homogeneous case, the energy-momentum tensor depends only on the energy density $\rho_{\mathrm{m}} \equiv-\bar{T}_{0}^{0}$ and the pressure $p_{\mathrm{m}} \equiv \bar{T}_{i}^{i} / 3$.

\subsection{Matter couplings and stability conditions}

To discuss the stability and determine the propagation speed of dark energy perturbations, one must also include quadratic terms that come from the matter action, because the latter depends on the gravitational degrees of freedom. In order to do so, we need to take into account that matter is minimally coupled to a metric $\check{g}_{\mu \nu}$ defined in eq. (2.8).

In unitary gauge, this definition reads

$$
\check{g}_{\mu \nu}=C_{\mathrm{m}}(t) g_{\mu \nu}+D_{\mathrm{m}}(t, N) \delta_{\mu}^{0} \delta_{\nu}^{0},
$$

with

$$
C_{\mathrm{m}}(t)=C_{\mathrm{m}}^{(\phi)}(\phi(t)), \quad D_{\mathrm{m}}(t, N)=\dot{\phi}^{2}(t) D_{\mathrm{m}}^{(\phi)}\left(\phi(t),-\dot{\phi}(t)^{2} / N^{2}\right) .
$$

Then, we introduce the parameters

$$
\alpha_{\mathrm{C}, \mathrm{m}} \equiv \frac{\dot{C}_{\mathrm{m}}}{2 H C_{\mathrm{m}}}, \quad \alpha_{\mathrm{D}, \mathrm{m}} \equiv \frac{D_{\mathrm{m}}}{C_{\mathrm{m}}-D_{\mathrm{m}}}, \quad \alpha_{\mathrm{X}, \mathrm{m}} \equiv-\frac{1}{2 C_{\mathrm{m}}} \frac{\partial D_{\mathrm{m}}}{\partial N},
$$

where the right-hand sides are evaluated on the background. The first two parameters in the above equations, $\alpha_{\mathrm{C}, \mathrm{m}}$ and $\alpha_{\mathrm{D}, \mathrm{m}}$, were introduced in Ref. [50].

Combining the quadratic action for matter with eq. (2.5), one can extract the dynamics of the gravitational scalar degree of freedom and the matter ones. The explicit calculation in the case of perfect fluids is presented in Appendix A. The absence of ghosts is guaranteed by the positivity of the matrix in front of the kinetic terms. For the gravitational scalar degree of freedom, this condition is given by

$$
\alpha \equiv \alpha_{\mathrm{K}}+6 \alpha_{\mathrm{B}}^{2}+3 \alpha_{\mathrm{D}, \mathrm{m}}^{\mathrm{eff}} \Omega_{\mathrm{m}} \geq 0,
$$

where $\Omega_{\mathrm{m}}$ is the standard (time-dependent) dimensionless density parameter,

$$
\Omega_{\mathrm{m}} \equiv \frac{\rho_{\mathrm{m}}}{3 M^{2} H^{2}}
$$

and we define the combination

$$
\alpha_{\mathrm{D}, \mathrm{m}}^{\mathrm{eff}} \equiv \alpha_{\mathrm{D}, \mathrm{m}}\left(1+\alpha_{\mathrm{X}, \mathrm{m}}\right)^{2}+\alpha_{\mathrm{X}, \mathrm{m}}\left(2+\alpha_{\mathrm{X}, \mathrm{m}}\right)+\frac{1}{2 C_{\mathrm{m}}} \frac{\partial^{2} D_{\mathrm{m}}}{\partial N^{2}} .
$$

Thus, the dependence on $X$ in the disformal coupling affects the ghost-free condition. 
Diagonalization of the kinetic matrix yields the following dispersion relation (see Appendix A for a generalization to multiple species)

$$
\left(\omega^{2}-c_{s}^{2} k^{2}\right)\left(\omega^{2}-c_{\mathrm{m}}^{2} k^{2}\right)=\lambda^{2} c_{s}^{2} \omega^{2} k^{2},
$$

where the parameter $\lambda^{2}$ on the right-hand side is defined as

$$
\lambda^{2} \equiv \frac{3}{\alpha c_{s}^{2}}\left[1+\left(1+\alpha_{\mathrm{D}, \mathrm{m}}\right) w_{\mathrm{m}}\right] \Omega_{\mathrm{m}}\left(\alpha_{\mathrm{H}}-\alpha_{\mathrm{X}, \mathrm{m}}\right)^{2} .
$$

This is the physically relevant parameter measuring the degree of KMM (as expected it is frame independent, see below). The $c_{s}^{2}$ appearing above is the sound speed of dark energy for $\lambda=0$, given by

$$
\begin{gathered}
c_{s}^{2}=-\frac{1}{\alpha}\left\{2\left(1+\alpha_{\mathrm{B}}\right)\left[\xi+\left(1+\alpha_{\mathrm{H}}\right) \frac{\dot{H}}{H^{2}}-\frac{\dot{\alpha}_{\mathrm{H}}}{H}\right]+2 \frac{\dot{\alpha}_{\mathrm{B}}}{H}\right. \\
\left.+3\left(1+\alpha_{\mathrm{H}}\right)^{2}\left[1+\left(1+\alpha_{\mathrm{D}, \mathrm{m}}\right) w_{\mathrm{m}}\right] \Omega_{\mathrm{m}}\right\},
\end{gathered}
$$

where for convenience we have defined

$$
\xi \equiv \alpha_{\mathrm{B}}\left(1+\alpha_{\mathrm{T}}\right)+\alpha_{\mathrm{T}}-\alpha_{\mathrm{M}}-\alpha_{\mathrm{H}}\left(1+\alpha_{\mathrm{M}}\right) .
$$

For $\alpha_{\mathrm{H}}=0$, this coincides with the parameter $\xi$ first defined in [50]. The above dispersion relation yields the two speeds of propagation

$$
c_{ \pm}^{2}=\frac{1}{2}\left\{c_{\mathrm{m}}^{2}+c_{s}^{2}\left(1+\lambda^{2}\right) \pm \sqrt{\left[c_{\mathrm{m}}^{2}+c_{s}^{2}\left(1+\lambda^{2}\right)\right]^{2}-4 c_{\mathrm{m}}^{2} c_{s}^{2}}\right\} .
$$

Equations (2.16) and (2.20) generalize the dispersion relations and speeds of propagation derived in $[27,59,68]$ for $\alpha_{\mathrm{X}, \mathrm{m}}=0$. The effect of KMM appears in the presence of the coupling $\lambda^{2} \neq 0$ and the propagation modes are mixed states of matter and scalar. In general, absence of gradient instabilities is guaranteed by the usual conditions $c_{ \pm}^{2} \geq 0$. Finally, when $\alpha_{\mathrm{X}, \mathrm{m}}=\alpha_{\mathrm{H}}$ we recover the usual results, i.e. $c_{+}^{2}=c_{s}^{2}$ and $c_{-}^{2}=c_{\mathrm{m}}^{2}$ for $c_{s}^{2}>c_{\mathrm{m}}^{2}$.

\section{$2.3(\partial \phi)^{2}$-dependent disformal transformations}

As mentioned earlier, there is some arbitrariness in the choice of the metric $g_{\mu \nu}$ that describes the gravitational sector. Let us thus see how the description is modified when the reference metric undergoes a disformal transformation, of the form

$$
g_{\mu \nu} \rightarrow \tilde{g}_{\mu \nu}=C^{(\phi)}(\phi) g_{\mu \nu}+D^{(\phi)}(\phi, X) \partial_{\mu} \phi \partial_{\nu} \phi
$$

which in unitary gauge corresponds to

$$
g_{\mu \nu} \rightarrow \tilde{g}_{\mu \nu}=C(t) g_{\mu \nu}+D(t, N) \delta_{\mu}^{0} \delta_{\nu}^{0} .
$$

The effect of this transformation on the ADM variables, on the background quantities and on the linear perturbations has been studied in detail in $[28,50]$. Here, we present the main consequences on the parametrization of the gravitational sector. 
In analogy with (2.12), it is convenient to introduce the dimensionless time-dependent parameters

$$
\alpha_{\mathrm{C}} \equiv \frac{\dot{C}}{2 H C}, \quad \alpha_{\mathrm{D}} \equiv \frac{D}{C-D}, \quad \alpha_{\mathrm{X}} \equiv-\frac{1}{2 C} \frac{\partial D}{\partial N},
$$

which characterize the conformal and disformal parts of the above metric transformation. ${ }^{4}$

Let us first see how the gravitational action (2.5) changes under the transformation (2.22). As shown in Ref. [28], the structure of the combination of the Horndeski and beyond Horndeski Lagrangians is preserved under a disformal transformation with an $X$-dependent disformal function $D$. Indeed, one can check that (2.5) maintains the same structure with the time-dependent coefficients in the action transforming as

$$
\tilde{M}^{2}=\frac{M^{2}}{C \sqrt{1+\alpha_{\mathrm{D}}}}
$$

and

$$
\begin{aligned}
\tilde{\alpha}_{\mathrm{K}} & =\frac{\alpha_{\mathrm{K}}+12 \alpha_{\mathrm{B}} \alpha_{\mathrm{CDX}}-6 \alpha_{\mathrm{CDX}}^{2}+3 \Omega_{\mathrm{m}}\left(1+\alpha_{\mathrm{X}, \mathrm{m}}\right) \alpha_{\mathrm{D}}^{\mathrm{eff}}}{\left(1+\alpha_{\mathrm{CDX}}\right)^{2}}, \\
\tilde{\alpha}_{\mathrm{B}} & =\frac{1+\alpha_{\mathrm{B}}}{1+\alpha_{\mathrm{CDX}}}-1, \\
\tilde{\alpha}_{\mathrm{M}} & =\frac{\alpha_{\mathrm{M}}-2 \alpha_{\mathrm{C}}}{1+\alpha_{\mathrm{C}}}-\frac{\dot{\alpha}_{\mathrm{D}}}{2 H\left(1+\alpha_{\mathrm{D}}\right)\left(1+\alpha_{\mathrm{C}}\right)}, \\
\tilde{\alpha}_{\mathrm{T}} & =\left(1+\alpha_{\mathrm{T}}\right)\left(1+\alpha_{\mathrm{D}}\right)-1 \\
\tilde{\alpha}_{\mathrm{H}} & =\frac{\alpha_{\mathrm{H}}-\alpha_{\mathrm{X}}}{1+\alpha_{\mathrm{X}}}
\end{aligned}
$$

where $\alpha_{\mathrm{CDX}} \equiv\left(1+\alpha_{\mathrm{C}}\right)\left(1+\alpha_{\mathrm{D}}\right)\left(1+\alpha_{\mathrm{X}}\right)-1$ and, in analogy with the definition $(2.15)$, we have introduced

$$
\alpha_{\mathrm{D}}^{\mathrm{eff}} \equiv \alpha_{\mathrm{D}}\left(1+\alpha_{\mathrm{X}}\right)^{2}+\alpha_{\mathrm{X}}\left(2+\alpha_{\mathrm{X}}\right)+\frac{1}{2 C} \frac{\partial^{2} D}{\partial N^{2}}
$$

We can use these transformations, which depend on the three arbitrary functions $\alpha_{\mathrm{C}}, \alpha_{\mathrm{D}}$ and $\alpha_{\mathrm{X}}$, to set to zero any three of the parameters $\tilde{\alpha}_{a}$ above.

Finally, the conformal and disformal coefficients associated with the respective matter Jordan frame metrics are modified according to

$$
\begin{aligned}
& \tilde{\alpha}_{\mathrm{D}, \mathrm{m}}=\frac{\alpha_{\mathrm{D}, \mathrm{m}}-\alpha_{\mathrm{D}}}{1+\alpha_{\mathrm{D}}}, \\
& \tilde{\alpha}_{\mathrm{C}, \mathrm{m}}=\frac{\alpha_{\mathrm{C}, \mathrm{m}}-\alpha_{\mathrm{C}}}{1+\alpha_{\mathrm{C}}}, \\
& \tilde{\alpha}_{\mathrm{X}, \mathrm{m}}=\frac{\alpha_{\mathrm{X}, \mathrm{m}}-\alpha_{\mathrm{X}}}{1+\alpha_{\mathrm{X}}} .
\end{aligned}
$$

These transformations can be straightforwardly extended to the case of different couplings to different species, for instance by simply replacing $\tilde{\alpha}_{\mathrm{D}, \mathrm{m}}$ by $\tilde{\alpha}_{\mathrm{D}, I}$ and $\alpha_{\mathrm{D}, \mathrm{m}}$ by $\alpha_{\mathrm{D}, I}$.

One can verify that the stability condition (2.13) is frame independent. In particular, $\alpha$ transforms as

$$
\tilde{\alpha}=\frac{\alpha}{\left(1+\alpha_{\mathrm{CDX}}\right)^{2}} .
$$

\footnotetext{
${ }^{4}$ As for the transformations in Secs. 2.1 and 2.2, we require $C>0$ and $\alpha_{\mathrm{D}}>-1$.
} 
It is also straightforward to check that all the propagation speeds, i.e. of tensor, scalar and matter fluctuations, transform in the same way and that their signs remain unchanged,

$$
\tilde{c}_{T}^{2}=\left(1+\alpha_{\mathrm{D}}\right) c_{T}^{2}, \quad \tilde{c}_{s}^{2}=\left(1+\alpha_{\mathrm{D}}\right) c_{s}^{2}, \quad \tilde{c}_{\mathrm{m}}^{2}=\left(1+\alpha_{\mathrm{D}}\right) c_{\mathrm{m}}^{2}
$$

Finally, using these expressions and those in [50] it is possible to show that the parameter $\lambda^{2}$ defined in eq. (2.17), which measures the degree of KMM, is frame independent as expected.

\section{Short-scale dynamics}

In this section we discuss the short-scale dynamics of cosmological perturbations. We assume universal coupling of matter species and, without loss of generality, minimal coupling. Thus, the action describing perturbations is given by (2.5), where the gravitational metric $g_{\mu \nu}$ is the Jordan frame metric. We focus on the scalar fluctuations and we employ the usual Stueckelberg procedure [20], $t \rightarrow t+\pi(t, \vec{x})$, to move from the unitary gauge to the Newtonian gauge, whose metric for a flat FLRW universe reads

$$
d s^{2}=-(1+2 \Phi) d t^{2}+a^{2}(1-2 \Psi) d \vec{x}^{2} .
$$

On short scales, the gradients of the scalar field $\phi$ support an oscillatory regime. In the presence of KMM, i.e. $\lambda^{2} \neq 0$, the oscillations are also shared by matter, even when matter is made of nonrelativistic species with no pressure gradients. We first describe these oscillations and their normal modes in the next subsection, while in Sec. 3.2 we discuss the late-time quasi-static regime occurring after the oscillations decay.

\subsection{Oscillatory regime and normal modes}

In this subsection, to describe matter we use a derivatively coupled scalar field $\sigma$, with action

$$
S_{\mathrm{m}}=\int d^{4} x \sqrt{-g} P(Y), \quad Y \equiv g^{\mu \nu} \partial_{\mu} \sigma \partial_{\nu} \sigma
$$

and we define the background energy density and pressure and the matter sound speed respectively as

$$
\rho_{\mathrm{m}}=-2 \dot{\sigma}_{0}^{2} P_{Y}(Y)-P(Y), \quad p_{\mathrm{m}}=P(Y), \quad c_{\mathrm{m}}^{2} \equiv \frac{P_{Y}}{P_{Y}-2 \dot{\sigma}_{0}^{2} P_{Y Y}} .
$$

We also introduce the energy density contrast and the velocity potential respectively as

$$
\delta_{\mathrm{m}} \equiv \frac{\delta \rho_{\mathrm{m}}}{\rho_{\mathrm{m}}}, \quad v_{\mathrm{m}} \equiv-\frac{\delta \sigma}{\dot{\sigma}_{0}} .
$$

For completeness, the full second-order actions describing the gravitational and matter sectors in Newtonian gauge in this case are given in Appendix B, eqs. (B.1) and (B.2).

To study the normal modes of oscillations we consider the kinetic limit, i.e. the limit where the spatial and time derivatives are larger than the expansion rate $H$. In this case, it is possible to find a redefinition of the metric perturbations that de-mixes the new metric variables from the scalar 
field $\pi$ and removes the higher derivative term from the gravitational action. This is explicitly given by [28]

$$
\begin{aligned}
\Phi_{E} & \equiv \frac{1+\alpha_{\mathrm{H}}}{1+\alpha_{\mathrm{T}}} \Phi+\left(\frac{1+\alpha_{\mathrm{M}}}{1+\alpha_{\mathrm{T}}}-\frac{1+\alpha_{\mathrm{B}}}{1+\alpha_{\mathrm{H}}}\right) H \pi-\frac{\alpha_{\mathrm{H}}}{1+\alpha_{\mathrm{T}}} \dot{\pi}, \\
\Psi_{E} & \equiv \Psi+\frac{\alpha_{\mathrm{H}}-\alpha_{\mathrm{B}}}{1+\alpha_{\mathrm{H}}} H \pi .
\end{aligned}
$$

Using these metric variables in the quadratic action and the definition (3.4) for $v_{\mathrm{m}}$, and writing explicitly only the terms that are quadratic in derivatives, neglecting those that are irrelevant in the kinetic limit, one finds the following action,

$$
\begin{aligned}
S_{\text {kinetic }}= & \int d^{4} x a^{3} M^{2}\left\{-3 \dot{\Psi}_{E}^{2}+\frac{1+\alpha_{\mathrm{T}}}{a^{2}}\left[\left(\nabla \Psi_{E}\right)^{2}-2 \nabla \Phi_{E} \nabla \Psi_{E}\right]\right. \\
& +\frac{\alpha H^{2}}{2\left(1+\alpha_{\mathrm{H}}\right)^{2}}\left[\left(1+\frac{c_{s}^{2}}{c_{\mathrm{m}}^{2}} \lambda^{2}\right) \dot{\pi}^{2}-c_{s}^{2} \frac{(\nabla \pi)^{2}}{a^{2}}\right] \\
& \left.+\frac{\rho_{\mathrm{m}}+p_{\mathrm{m}}}{2 c_{\mathrm{m}}^{2} M^{2}}\left[\dot{v}_{\mathrm{m}}^{2}-c_{\mathrm{m}}^{2} \frac{\left(\nabla v_{\mathrm{m}}\right)^{2}}{a^{2}}+\frac{2 \alpha_{\mathrm{H}}}{1+\alpha_{\mathrm{H}}} \dot{v}_{\mathrm{m}} \dot{\pi}\right]\right\},
\end{aligned}
$$

where $\lambda^{2}$ is the parameter encoding KMM, defined in eq. (2.17). Since here we are using the Jordan frame metric, where $\alpha_{\mathrm{D}, \mathrm{m}}=\alpha_{\mathrm{X}, \mathrm{m}}=0$, its definition reads

$$
\lambda^{2}=\frac{3}{\alpha c_{s}^{2}} \alpha_{\mathrm{H}}^{2}\left(1+w_{\mathrm{m}}\right) \Omega_{\mathrm{m}}
$$

so that $\lambda$ is proportional to $\alpha_{\mathrm{H}}$. Notice in the third line the presence of a kinetic coupling between the scalar and matter fields, $\dot{v}_{\mathrm{m}} \dot{\pi}$, proportional to $\alpha_{\mathrm{H}}$.

Moreover, at this order in derivatives the dynamics of $\pi$ and $v_{\mathrm{m}}$ is decoupled from that of $\Phi_{E}$ and $\Psi_{E}$ and we can study them separately. To simplify the analysis, we introduce the canonically normalized fields

$$
\pi_{\mathrm{c}} \equiv \frac{H M \alpha^{1 / 2}}{1+\alpha_{\mathrm{H}}} \pi, \quad v_{\mathrm{c}} \equiv\left(\frac{\rho_{\mathrm{m}}+p_{\mathrm{m}}}{c_{\mathrm{m}}^{2}}\right)^{1 / 2} v_{\mathrm{m}},
$$

and we neglect the expansion of the universe, which is irrelevant in the kinetic limit. Then the dynamics is described by the Lagrangian

$$
\mathcal{L}=\frac{1}{2}\left\{\left(1+\frac{c_{s}^{2}}{c_{\mathrm{m}}^{2}} \lambda^{2}\right) \dot{\pi}_{\mathrm{c}}^{2}-c_{s}^{2}\left(\nabla \pi_{\mathrm{c}}\right)^{2}+\dot{v}_{\mathrm{c}}^{2}-c_{\mathrm{m}}^{2}\left(\nabla v_{\mathrm{c}}\right)^{2}+2 \frac{c_{s}}{c_{\mathrm{m}}} \lambda \dot{v}_{\mathrm{c}} \dot{\pi}_{\mathrm{c}}\right\}
$$

In Fourier space, this gives the coupled system of equations

$$
\frac{d^{2}}{d t^{2}}\left(\begin{array}{l}
\pi_{c} \\
v_{c}
\end{array}\right)+k^{2}\left(\begin{array}{cc}
c_{s}^{2} & -\lambda c_{s} c_{\mathrm{m}} \\
-\lambda c_{s}^{3} / c_{\mathrm{m}} & c_{\mathrm{m}}^{2}+\lambda^{2} c_{s}^{2}
\end{array}\right)\left(\begin{array}{l}
\pi_{c} \\
v_{c}
\end{array}\right)=0,
$$

with normal modes

$$
\left(\begin{array}{cc}
c_{s}^{3} \lambda / c_{\mathrm{m}} & c_{-}^{2}-c_{s}^{2} \\
-c_{s}^{3} \lambda / c_{\mathrm{m}} & c_{s}^{2}-c_{+}^{2}
\end{array}\right)\left(\begin{array}{l}
\pi_{c} \\
v_{c}
\end{array}\right)
$$

where $c_{ \pm}^{2}$ are the eigenvalues of the system, given by eq. (2.20). 
As an example relevant for late-time cosmology, we consider the case where matter is described by a non-relativistic fluid (for instance CDM) with $w_{\mathrm{m}}=0$ and $c_{\mathrm{m}}^{2}=0$. Going back to standard normalization before setting $c_{\mathrm{m}}^{2}=0$, the eigenmodes and respective eigenvalues of the system are

$$
\begin{aligned}
& X_{-}=v_{\mathrm{m}}+\pi \frac{\alpha_{\mathrm{H}}}{1+\alpha_{\mathrm{H}}}, \quad c_{-}^{2}=c_{\mathrm{m}}^{2}=0, \\
& X_{+}=\pi-v_{\mathrm{m}} \lambda^{2} \frac{1+\alpha_{\mathrm{H}}}{\alpha_{\mathrm{H}}},
\end{aligned}
$$

with $\lambda^{2}=3 \alpha_{\mathrm{H}}^{2} \Omega_{\mathrm{m}} /\left(\alpha c_{s}^{2}\right)$. While $X_{+}$displays oscillations with frequency $\omega= \pm i c_{+} k$, the speed of the fluctuations of $X_{-}$vanishes as that of matter.

\subsection{Quasi-static regime}

Here we stick to the case where matter is non-relativistic, i.e. $p_{\mathrm{m}}=c_{\mathrm{m}}=0$, which applies to matter in late-time cosmology. When including the Hubble expansion, we expect the oscillations of $X_{+}$ to get damped [52]. In the absence of the oscillatory mode $X_{+}$, the time evolution is dominated by the Hubble friction and time derivatives are of the order of the Hubble rate $H$. This is the quasi-static regime. We leave for the App. $\mathrm{C}$ the discussion of how this regime is reached in the cosmological evolution.

In this case, focussing on the short-scale limit $k \gg k_{+}$, where $k_{+}$denotes the sound horizon scale of the oscillating mode,

$$
k_{+} \equiv \frac{a H}{c_{+}}=\frac{a H}{c_{s} \sqrt{1+\lambda^{2}}},
$$

and neglecting oscillations, the second-order action in Newtonian gauge becomes ${ }^{5}$

$$
S=\int d^{4} x a^{3} M^{2}\left\{\frac{1+\alpha_{\mathrm{T}}}{a^{2}}\left[\left(\nabla \Psi_{E}\right)^{2}-2 \nabla \Phi_{E} \nabla \Psi_{E}\right]-\frac{\alpha H^{2} c_{s}^{2}}{2\left(1+\alpha_{\mathrm{H}}\right)^{2}} \frac{(\nabla \pi)^{2}}{a^{2}}-\Phi \frac{\delta \rho_{\mathrm{m}}}{M^{2}}\right\} .
$$

Variation of the above action with respect to $\Phi_{E}$ yields a Poisson-like equation for $\Psi_{E}$,

$$
\frac{\nabla^{2} \Psi_{E}}{a^{2}}=\frac{3}{2} H^{2} \Omega_{\mathrm{m}} \frac{\delta_{\mathrm{m}}}{1+\alpha_{\mathrm{H}}} .
$$

In the above limit, also the scalar field fluctuations $\pi$ satisfy a Poisson-like equation. To derive it, one can vary the action (3.16) with respect to $\pi$, taking into account that $\Phi_{E}$ and $\Psi_{E}$ depend on $\pi$ through the expressions (3.5). Using eq. (3.17), $\Phi_{E}=\Psi_{E}$, the definition of $\delta_{\mathrm{m}}$, eq. (3.4), and the continuity equation for matter,

$$
\dot{\delta}_{\mathrm{m}}=-\frac{\nabla^{2} v_{\mathrm{m}}}{a^{2}}
$$

one obtains

$$
\frac{\nabla^{2} \pi}{a^{2}}=\frac{3 H \Omega_{\mathrm{m}}}{c_{s}^{2} \alpha}\left[\left(\xi-\frac{\dot{\alpha}_{\mathrm{H}}}{H}\right) \delta_{\mathrm{m}}+\frac{\alpha_{\mathrm{H}}\left(1+\alpha_{\mathrm{H}}\right)}{H} \frac{\nabla^{2} v_{\mathrm{m}}}{a^{2}}\right],
$$

\footnotetext{
${ }^{5}$ To get the last term one can replace in eq. (B.2) the time derivative of the field fluctuation $\delta \dot{\sigma}$ by its density fluctuation $\delta \rho_{\mathrm{m}}$, using the expression

$$
\delta \rho_{\mathrm{m}}=\left(1+\frac{1}{c_{\mathrm{m}}^{2}}\right)\left(\rho_{\mathrm{m}}+p_{\mathrm{m}}\right)\left(\frac{\delta \dot{\sigma}}{\dot{\sigma}_{0}}-\Phi\right)
$$

valid for finite $c_{\mathrm{m}}^{2}$, and subsequently set $c_{\mathrm{m}}^{2}=0$.
} 
where we remind the reader that $\xi \equiv \alpha_{\mathrm{B}}\left(1+\alpha_{\mathrm{T}}\right)+\alpha_{\mathrm{T}}-\alpha_{\mathrm{M}}-\alpha_{\mathrm{H}}\left(1+\alpha_{\mathrm{M}}\right)$ (see eq. (2.19)). Notice the presence of the last term on the right-hand side, proportional to the matter velocity, which stems from the KMM. Indeed, by using the definition of the "+" eigenmode $X_{+}$, eq. (3.13), this equation can be rewritten as

$$
\frac{\nabla^{2} X_{+}}{a^{2}}=\frac{3 H \Omega_{\mathrm{m}}}{c_{s}^{2} \alpha}\left(\xi-\frac{\dot{\alpha}_{\mathrm{H}}}{H}\right) \delta_{\mathrm{m}},
$$

which shows that after the oscillating regime ends, $X_{+}$(and not $\pi$ ) satisfies a Poisson-like constraint equation.

Let us now derive the constraint equations for $\Psi$ and $\Phi$. We can rewrite equation (3.17) in terms of $\Psi$ using the definition of $\Psi_{E}$, eq. (3.5), and eq. (3.19). We can then use $\Phi_{E}=\Psi_{E}$ and solve eqs. (3.17), (3.19) and its derivative to find an equation for $\Phi$. This yields

$$
\begin{aligned}
\frac{\nabla^{2} \Psi}{a^{2}} & =\frac{3}{2} H^{2} \Omega_{\mathrm{m}} \mu_{\Psi} \delta_{\mathrm{m}}+\lambda^{2}\left(\frac{\alpha_{\mathrm{B}}}{\alpha_{\mathrm{H}}}-1\right) H \frac{\nabla^{2} v_{\mathrm{m}}}{a^{2}}, \\
\frac{\nabla^{2} \Phi}{a^{2}} & =\frac{3}{2} H^{2} \Omega_{\mathrm{m}} \mu_{\Phi} \delta_{\mathrm{m}}+\gamma H \frac{\nabla^{2} v_{\mathrm{m}}}{a^{2}},
\end{aligned}
$$

where $\mu_{\Psi}$ and $\mu_{\Phi}$ are defined as

$$
\begin{aligned}
& \mu_{\Psi} \equiv \frac{1}{1+\alpha_{\mathrm{H}}}\left[1+\frac{2\left(\alpha_{\mathrm{B}}-\alpha_{\mathrm{H}}\right)}{c_{s}^{2} \alpha}\left(\xi-\frac{\dot{\alpha}_{\mathrm{H}}}{H}\right)\right], \\
& \mu_{\Phi} \equiv \frac{1}{\left(1+\lambda^{2}\right)\left(1+\alpha_{\mathrm{H}}\right)^{2}}\left\{c_{T}^{2}+\frac{2 \xi}{c_{s}^{2} \alpha}\left(\xi-\frac{\dot{\alpha}_{\mathrm{H}}}{H}\right)+a \alpha_{\mathrm{H}}\left(1+\alpha_{\mathrm{H}}\right)\left[\frac{2}{a H c_{s}^{2} \alpha}\left(\xi-\frac{\dot{\alpha}_{\mathrm{H}}}{H}\right)\right]\right\},
\end{aligned}
$$

and $\gamma$ is defined as

$$
\gamma \equiv \frac{d \ln \left(1+\lambda^{2}\right)}{d \ln a}
$$

The parameters $\mu_{\Psi}$ and $\mu_{\Phi}$ represent modifications of the Poisson law, respectively for $\Psi$ and $\Phi$, and are equal to one in the standard case. The last term on the right-hand side of eqs. (3.21) and (3.22) proportional to the Laplacian of the matter velocity potential vanishes in the absence of KMM.

Equation (3.22), together with the continuity equation (3.18) and the Euler equation,

$$
\dot{v}_{\mathrm{m}}=-\Phi
$$

can be used to derive a closed second-order equation for the matter density contrast in the quasistatic limit. Indeed, taking the time derivative of the continuity equation, and plugging in the latter the Euler equation and eq. (3.22), one obtains [58],

$$
\ddot{\delta}_{\mathrm{m}}+(2+\gamma) H \dot{\delta}_{\mathrm{m}}=\frac{3}{2} H^{2} \Omega_{\mathrm{m}} \mu_{\Phi} \delta_{\mathrm{m}} .
$$

A comment on this equation is in order here. For $\alpha_{\mathrm{H}}=0$, the friction term vanishes, $\gamma=0$, and the strength of gravitational clustering is modified by [35]

$$
\mu_{\Phi}=c_{T}^{2}+\frac{2 \xi^{2}}{c_{s}^{2} \alpha}, \quad\left(\alpha_{\mathrm{H}}=0\right)
$$


which, for $c_{T}^{2} \geq 1,{ }^{6}$ is always larger than one. Thus, the exchange of the fifth force tends to enhance gravity on small scales $[30,35,41,50]$. On the contrary, in the presence of KMM $\mu_{\Phi}-c_{T}^{2}$ can be negative, corresponding to a repulsive scalar fifth-force, thus weakening gravity. Moreover, the last term on the right-hand side of (3.22) can act as a friction term for structure formation. This results in a suppression of clustering, even for a $\Lambda$ CDM background evolution. We will see an explicit example below.

For completeness and comparison with observations, we provide here also the expression of the Weyl potential, obtained by summing eqs. (3.21) and (3.22),

$$
\frac{1}{a^{2} H^{2}} \nabla^{2}(\Phi+\Psi)=\frac{3}{2} \Omega_{\mathrm{m}}\left(\mu_{\Psi}+\mu_{\Phi}\right) \delta_{\mathrm{m}}+\left[\left(1-\frac{\alpha_{\mathrm{B}}}{\alpha_{\mathrm{H}}}\right) \lambda^{2}-\gamma\right] \frac{\dot{\delta}_{\mathrm{m}}}{H}
$$

where we have used the continuity equation to replace the velocity $v_{\mathrm{m}}$ by $\dot{\delta}_{\mathrm{m}}$. Note that for $\alpha_{\mathrm{H}}=0$, the equations in this section reduce to their analogous expressions derived for instance in [35].

\section{Observational signatures of Kinetic Matter Mixing}

In this section we discuss the effects of KMM on the power spectrum of the matter density contrast and on the CMB. In particular, we compute the comoving matter density contrast, defined as

$$
\Delta_{\mathrm{m}} \equiv \delta_{\mathrm{m}}-3 H v_{\mathrm{m}},
$$

where $\delta_{\mathrm{m}}$ and $v_{\mathrm{m}}$ are in Newtonian gauge. For the CMB we focus on the lensing potential and the temperature fluctuations.

The observables are computed using COOP [47], which solves linear perturbations in Newtonian gauge and in the Jordan frame, assuming minimal coupling of all matter species. In the $\Lambda$ CDM case, COOP evolves $\Psi, \Psi_{N_{e}} \equiv d \Psi / d N_{e}$ and matter perturbations, where $N_{e} \equiv \ln a$ is the program time variable. The detailed algorithm and equations can be found in Ref. [71]. To describe deviations from $\Lambda$ CDM using the Effective Theory of Dark Energy, COOP evolves two additional variables, $\mu \equiv H \pi$ and $\mu_{N_{e}} \equiv d \mu / d N_{e}$. In the Jordan frame, only the metric perturbations are coupled to $\mu$ and $\mu_{N_{e}}$. The evolution equations of $\Psi_{N_{e}}$ and $\mu_{N_{e}}$ are obtained by eliminating $\Phi$ from eqs. (111)-(113) in Ref. [10]. For numeric stability, COOP combines the energy conservation equation and the pressure equation, respectively eqs. (109) and (112) of Ref. [10], such that the evolution equation of $\Psi_{N_{e}}$ has a traceless source, i.e. it is of the form $d \Psi_{N_{e}} / d N_{e}=\ldots+\left(\delta p_{\mathrm{m}}-\right.$ $\left.\frac{1}{3} \delta \rho_{\mathrm{m}}\right) /\left(2 M^{2}\right)$. See Ref. [71] for more details on this technique. Once the linear perturbations are solved, COOP computes CMB power spectra using a line-of-sight integral [72,73]. Matter power spectra are computed via a gauge transformation from the Newtonian to the CDM rest-frame synchronous gauge.

For the cosmological parameters we use the Planck TT+lowP parameters [67]. In particular, we assume a physical density of baryons and CDM respectively given by $\Omega_{b, 0} h^{2}=0.02222$ and $\Omega_{c, 0} h^{2}=0.1197$, we fix the acoustic scale at recombination as $\theta=1.04085 \times 10^{-2}$, the amplitude of scalar primordial fluctuations $A_{s}=2.2 \times 10^{-9}$, the scalar spectral tilt $n_{s}=0.9655$ and the reionization optical depth $\tau=0.078$. We assume that the background expansion history is the same as in $\Lambda$ CDM. This implies that $h=67.31$ and $\Omega_{\mathrm{m}, 0}=0.315$. Initial conditions are taken to be adiabatic (see e.g. [10]).

\footnotetext{
${ }^{6}$ Cosmic rays observations put tight constraints on a propagation speed $c_{T}^{2}<1$ [69]. Another lower bound can be put from binary pulsar orbital periods [70].
} 
To focus on the effects of KMM, we set

$$
\alpha_{\mathrm{B}}=\alpha_{\mathrm{M}}=\alpha_{\mathrm{T}}=0 .
$$

Moreover, we parametrize the time dependence of $\alpha_{\mathrm{K}}$ and $\alpha_{\mathrm{H}}$ as

$$
\alpha_{\mathrm{K}}=\alpha_{\mathrm{K}, 0} \frac{\Omega_{\mathrm{DE}}(t)}{\Omega_{\mathrm{DE}, 0}}, \quad \alpha_{\mathrm{H}}=\alpha_{\mathrm{H}, 0} \frac{\Omega_{\mathrm{DE}}(t)}{\Omega_{\mathrm{DE}, 0}},
$$

where $\Omega_{\mathrm{DE}}$ is the fractional energy density of dark energy, defined as $\Omega_{\mathrm{DE}} \equiv 1-\sum_{I} \Omega_{I}$, where the sum is over all matter species (baryons, photons, neutrinos and CDM).

For the sake of clarity, in the following discussion we will simplify the above parametrization and consider only baryons and CDM in the matter sector. This is justified by the fact that according to this parametrization, the effects of dark energy become relevant only at late time. However, we stress that the numerical calculation performed with COOP contains the full matter sector, including (massless) neutrinos. Under these simplifying assumptions the background expansion history becomes

$$
H^{2}=H_{0}^{2}\left[\Omega_{\mathrm{m} 0} a^{-3}+1-\Omega_{\mathrm{m} 0}\right] .
$$

Moreover, in this case the speed of scalar fluctuations (see eq. (2.18)) simplifies to

$$
c_{s}^{2}=\frac{\alpha_{\mathrm{H}}\left[2+3 \Omega_{\mathrm{m}}\left(1-\alpha_{\mathrm{H}}\right)\right]}{\alpha_{\mathrm{K}}} .
$$

Requiring the absence of ghosts $(\alpha>0$, see definition in eq. (2.13)) and gradient instabilities, respectively implies that

$$
\alpha_{\mathrm{K}} \geq 0, \quad 0 \leq \alpha_{\mathrm{H}} \leq 1+\frac{2}{3 \Omega_{\mathrm{m}}} .
$$

In the following we set the current value of $\alpha_{\mathrm{K}}$ to unity, $\alpha_{\mathrm{K}, 0}=1$ and we plot the effect of $\alpha_{\mathrm{H}}$ in terms of four different values of this parameter today, i.e. $\alpha_{\mathrm{H}, 0}=0.06,0.12,0.24$ and 0.48 , which are always in the stability window (4.6). Note that to avoid that scalar fluctuations become superluminal in the past we must require

$$
\alpha_{\mathrm{H}} \leq \frac{1}{5} \alpha_{\mathrm{K}}
$$

Just for the purpose of illustration, in the next two subsections we ignore constraints from superluminality, as we need large values of $\alpha_{\mathrm{H}}$ to better visualise the effects on the observables.

\subsection{Matter power spectrum}

On short scales, increasing $\alpha_{\mathrm{H}, 0}$ suppresses the power spectrum of matter fluctuations, shown as a function of $k$ in Fig. 1. On these scales we can neglect the velocity potential in the definition of the comoving matter density contrast, eq. (4.1), which reduces to $\delta_{\mathrm{m}}$ in the Newtonian gauge, $\Delta_{\mathrm{m}} \approx \delta_{\mathrm{m}}$. Moreover, to understand the power suppression we can apply the quasi-static approximation, i.e. eq. (3.27). Specializing to the case with only nonvanishing $\alpha_{\mathrm{K}}$ and $\alpha_{\mathrm{H}}$ and using the time parametrization above, $\mu_{\Phi}$ and $\gamma$ in eq. (3.27), defined in eqs. (3.24) and (3.25), become

$$
\mu_{\Phi}=1-\gamma, \quad \gamma=-\Omega_{\mathrm{m}} \frac{9 \alpha_{\mathrm{H}}\left(2-4 \Omega_{\mathrm{m}}-3 \Omega_{\mathrm{m}}^{2}\right)}{\left(2+3 \Omega_{\mathrm{m}}\right)\left[2+3\left(1-\alpha_{\mathrm{H}}\right) \Omega_{\mathrm{m}}\right]} .
$$



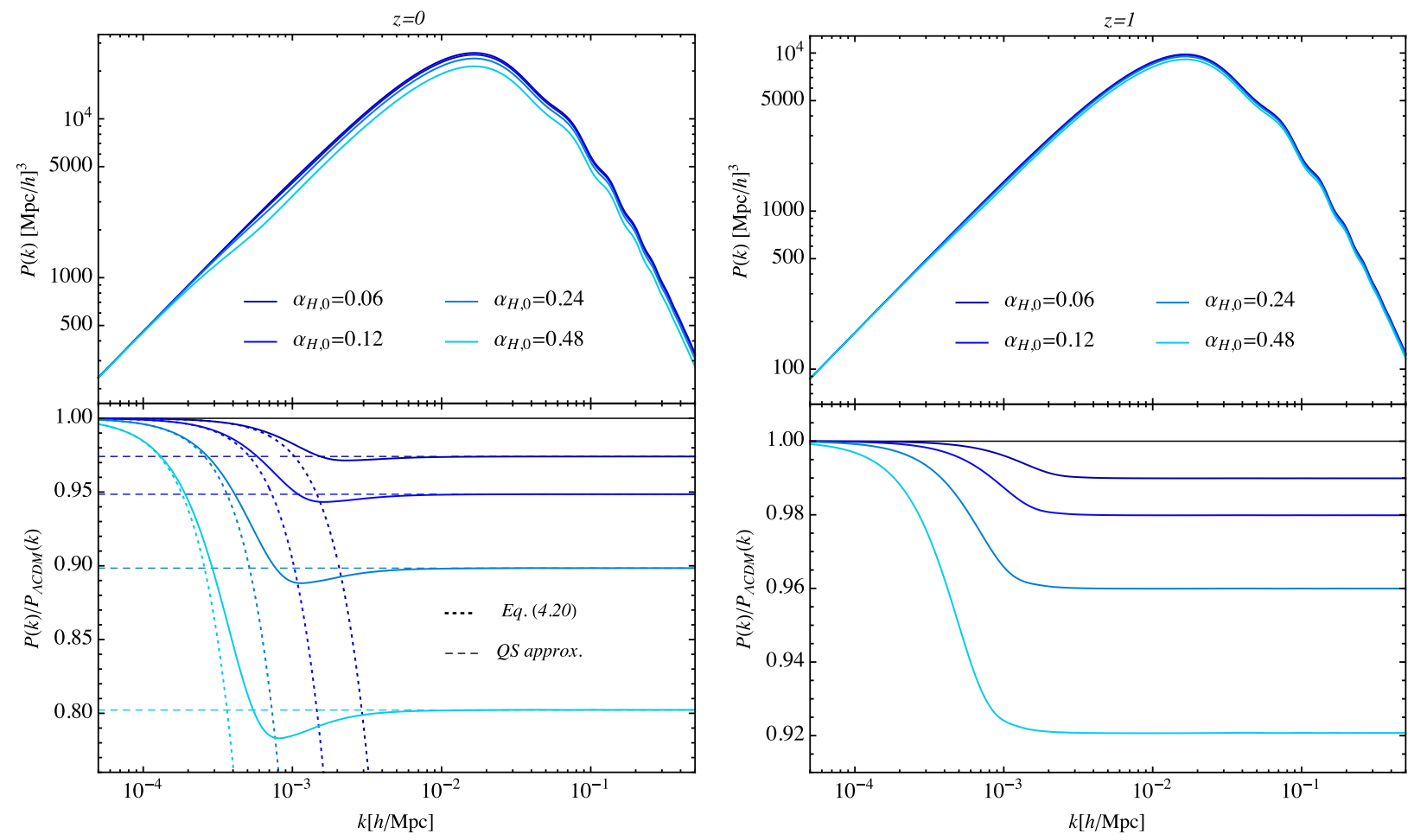

Figure 1: Effect of KMM on the matter power spectrum for four different values of $\alpha_{\mathrm{H}}$ today, i.e. $\alpha_{\mathrm{H}, 0}=0.06$, $0.12,0.24$ and 0.48 , at redshift $z=0$ (left panel) and $z=1$ (right panel). The lower plots display the ratio of these power spectra with the respective spectra for $\alpha_{\mathrm{H}}=0$. For comparison, the dashed and dotted lines in the left lower panel respectively show the quasi-static approximation and the perturbative solution of eq. (4.20).

The friction term $\gamma$ as a function of redshift is plotted in Fig. 2. It starts positive and changes sign only recently, when $\Omega_{\mathrm{m}}=(\sqrt{10}-2) / 3 \simeq 0.39$. In particular, during matter domination it behaves as

$$
\gamma=\frac{9}{5} \alpha_{\mathrm{H}}+\mathcal{O}\left(\Omega_{\mathrm{DE}}^{2}\right)
$$

where we have expanded in $\Omega_{\mathrm{DE}}$. Thus, $\gamma$ suppresses the power spectrum with respect to the $\Lambda \mathrm{CDM}$ case and the effect is linear in $\alpha_{\mathrm{H}}$. The modification of the Poisson equation has an analogous effect: $\mu_{\Phi}$ starts smaller than unity decreasing the strength of gravity, and gets larger than one only when $\gamma$ changes sign. This has again the cumulative effect of suppressing the power spectrum with respect to the $\Lambda \mathrm{CDM}$ case. We have checked that eq. (3.27), with $\gamma$ and $\mu_{\Phi}$ given above, reproduces the suppression observed in Fig. 1.

Corrections to the quasi-static approximation are expected to be of the order $\mathcal{O}\left(k_{+}^{2} / k^{2}\right)$, where $k_{+}$is the sound horizon scale defined in eq. (3.14). ${ }^{7}$ Thus, on larger scales this approximation fails to reproduce the correct spectrum, as shown in the figure. However, we can find an integral solution for the density perturbation on the largest scales by solving the Einstein and scalar field equations perturbatively in $\alpha_{\mathrm{H}}$ (while keeping the exact dependence on $\alpha_{\mathrm{K}}$ to avoid inconsistencies [74]). For

\footnotetext{
${ }^{7}$ The sound horizon scale is $k_{+} \simeq 8.1,5.8,4.2$ and $3.1 \times 10^{-4} h / \mathrm{Mpc}$ at redshift $z=0$ and $k_{+} \simeq 5.9,4.2,3.0$ and $2.2 \times 10^{-4} h / \mathrm{Mpc}$ at redshift $z=1$, respectively for $\alpha_{\mathrm{H}, 0}=0.06,0.12,0.24$ and 0.48 .
} 


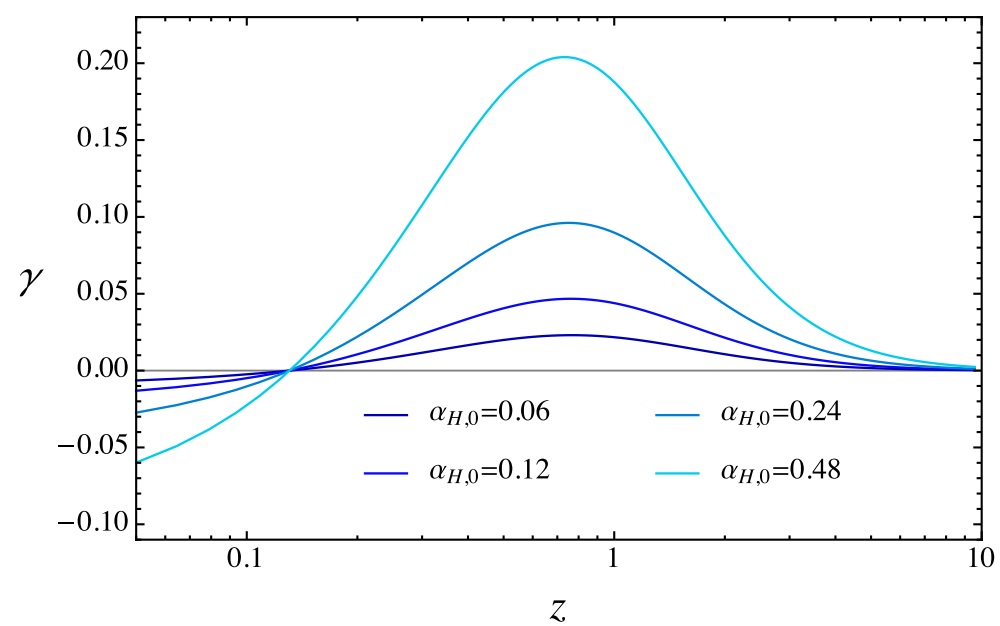

Figure 2: Friction term $\gamma$ given in eq. (4.8), as a function of redshift.

the parametrization chosen in this section, these equations read

$$
\begin{aligned}
-\frac{k^{2}}{a^{2}} \Psi+\dot{H}\left(\Delta_{\mathrm{m}}+\Delta_{\mathrm{DE}}\right) & =0, \\
\Phi-\Psi & =\alpha_{\mathrm{H}}(\dot{\pi}-\Phi), \\
\ddot{\Psi}+H(3 \dot{\Psi}+\dot{\Phi})+\left(2 \dot{H}+3 H^{2}\right) \Phi & =0, \\
\dot{\Delta}_{\mathrm{DE}} & =-\alpha_{\mathrm{H}} \frac{k^{2}}{a^{2}} \frac{H}{\dot{H}}(\dot{\pi}-\Phi),
\end{aligned}
$$

where we have used the background Friedmann equations and the comoving energy density contrast associated to dark energy, $\Delta_{\mathrm{DE}}$, is defined as

$$
\Delta_{\mathrm{DE}} \equiv-\frac{\alpha_{\mathrm{K}}}{2} H^{2}(\dot{\pi}-\Phi)-\alpha_{\mathrm{H}} \frac{k^{2}}{a^{2}}(\Psi+H \pi) .
$$

Equation (4.10) has been obtained from combining the " 00 " and " $0 i$ " scalar components of the Einstein equations, eqs. (4.11) and (4.12) are respectively the traceless and trace part of the " $i j$ " scalar components of the Einstein equations and eq. (4.13) is the evolution equation of $\pi .^{8}$ (The evolution equations for the matter density contrast $\Delta_{\mathrm{m}}$ is automatically satisfied by these equations.)

In the absence of KMM, i.e. for $\alpha_{\mathrm{H}}=0$, eqs. (4.10)-(4.13) are solved by the standard $\Lambda \mathrm{CDM}$ solution with adiabatic initial conditions [10], i.e.

$$
\Phi=-\dot{\epsilon}, \quad \Psi=H \epsilon-\zeta_{0}, \quad \Delta_{\mathrm{m}}=\Delta_{\mathrm{m}}^{(0)} \equiv \frac{k^{2}}{a^{2}} \frac{H \epsilon-\zeta_{0}}{\dot{H}}, \quad \Delta_{\mathrm{DE}}=0, \quad\left(\alpha_{\mathrm{H}}=0\right)
$$

where $\zeta_{0}$ is the (conserved) comoving curvature perturbation on super-Hubble scales and $\epsilon$ is defined as

$$
\epsilon \equiv \frac{\zeta_{0}}{a} \int a d t
$$

\footnotetext{
${ }^{8}$ The complete Einstein equations can be found in [10].
} 
Notice that, for $\alpha_{\mathrm{H}}=0, \pi=-\epsilon$ and thus the combination $\dot{\pi}-\Phi$ vanishes. Hence, the right-hand sides of eqs. (4.11) and (4.13) vanish also at first order in $\alpha_{\mathrm{H}}$ and eq. (4.15) keeps being a solution of the above equations.

The combination $\dot{\pi}-\Phi$ does not vanish at first order in $\alpha_{\mathrm{H}}$. Using in eq. (4.14) that $\Delta_{\mathrm{DE}}=0$ at this order, one obtains

$$
\dot{\pi}-\Phi=2 \zeta_{0} \frac{k^{2}}{a^{2} H^{2}} \frac{\alpha_{\mathrm{H}}}{\alpha_{\mathrm{K}}}+\mathcal{O}\left(\alpha_{\mathrm{H}}^{2}\right) .
$$

Thus, deviations from $\Lambda \mathrm{CDM}$ arise at second-order in $\alpha_{\mathrm{H}}$, as the backreaction effect of $\pi$ on gravity. This is similar to what happens in the context of the Ghost Condensate, where the mixing of the scalar fluctuations with gravity gives rise to a Jeans-like instability also on a $\Lambda$ CDM background $[5,75]$. It is now straightforward to find the solution for $\Delta_{\mathrm{m}}$ at this order in $\alpha_{\mathrm{H}}$, by replacing the second-order solution for $\Psi$ and $\Delta_{\mathrm{DE}}$ in eq. (4.10). The former can be derived by solving eq. (4.12) after replacing $\Phi$ from eq. (4.11). This yields

$$
\Psi=H \epsilon-\zeta_{0}+2 \zeta_{0} \frac{\alpha_{\mathrm{H}}^{2}}{\alpha_{\mathrm{K}}} \frac{k^{2}}{a^{2} H^{2}}\left(1-a H^{5} \int \frac{d t}{a H^{4}}\right)+\mathcal{O}\left(\alpha_{\mathrm{H}}^{3}\right) .
$$

The latter can be derived from eq. (4.13), which yields

$$
\Delta_{\mathrm{DE}}=-2 \zeta_{0} \frac{\alpha_{\mathrm{H}}^{2}}{\alpha_{\mathrm{K}}} \frac{k^{4}}{a^{4}} \frac{a H^{2}}{\dot{H}} \int \frac{d t}{a H^{3}}+\mathcal{O}\left(\alpha_{\mathrm{H}}^{3}\right) .
$$

Thus, one obtains

$$
\Delta_{\mathrm{m}}=\Delta_{\mathrm{m}}^{(0)}\left[1-2 a H^{2} \frac{\alpha_{\mathrm{H}}^{2}}{\alpha_{\mathrm{K}}} \frac{k^{2}}{a^{2}}\left(\int \frac{d t}{a H^{3}}-H \int \frac{d t}{a H^{4}}\right)\left(1-\frac{H}{a} \int a d t\right)^{-1}+\mathcal{O}\left(\alpha_{\mathrm{H}}^{3}\right)\right] .
$$

Notice that this solution breaks down on small scales because the quasi-static limit assumes $\alpha_{\mathrm{H}} \neq 0$.

On very large scales, i.e. for

$$
\begin{aligned}
k \lesssim k_{*} & \equiv \frac{\sqrt{\alpha_{\mathrm{K}}}}{\sqrt{2} \alpha_{\mathrm{H}}} \frac{a}{H}\left(1-\frac{H}{a} \int a d t\right)^{1 / 2}\left(a \int \frac{d t}{a H^{3}}-H a \int \frac{d t}{a H^{4}}\right)^{-1 / 2} \\
& \simeq \frac{\sqrt{\alpha_{\mathrm{K}, 0}}}{\alpha_{\mathrm{H}, 0}} \times 5.4 \times 10^{-4} h / \mathrm{Mpc},
\end{aligned}
$$

the power spectrum is unmodified by KMM, although this restricts only to the case where the background expansion is that of $\Lambda$ CDM. On intermediate scales, $k_{*} \lesssim k \lesssim k_{+}$, the power spectrum drops as $k^{2}$ due to the second term on the right-hand side of eq. (4.20).

\subsection{Cosmic Microwave Background}

In Fig. 3, on the left panel we plot the angular power spectrum of the CMB lensing potential, defined as $[76]^{9}$

$$
\phi(\hat{n})=-\int_{0}^{z_{*}} \frac{d z}{H(z)} \frac{\chi\left(z_{*}\right)-\chi(z)}{\chi\left(z_{*}\right) \chi(z)}[\Phi(\chi \hat{n}, z)+\Psi(\chi \hat{n}, z)],
$$

where $\chi \equiv \int_{0}^{z} d z / H(z)$ is the conformal distance and $z_{*}$ denotes the redshift of last scattering. On the right panel, we plot the angular power spectrum of the CMB anisotropies as a function of the

\footnotetext{
${ }^{9}$ This is not to be confused with the scalar field $\phi$ introduced in Sec. 2.
} 

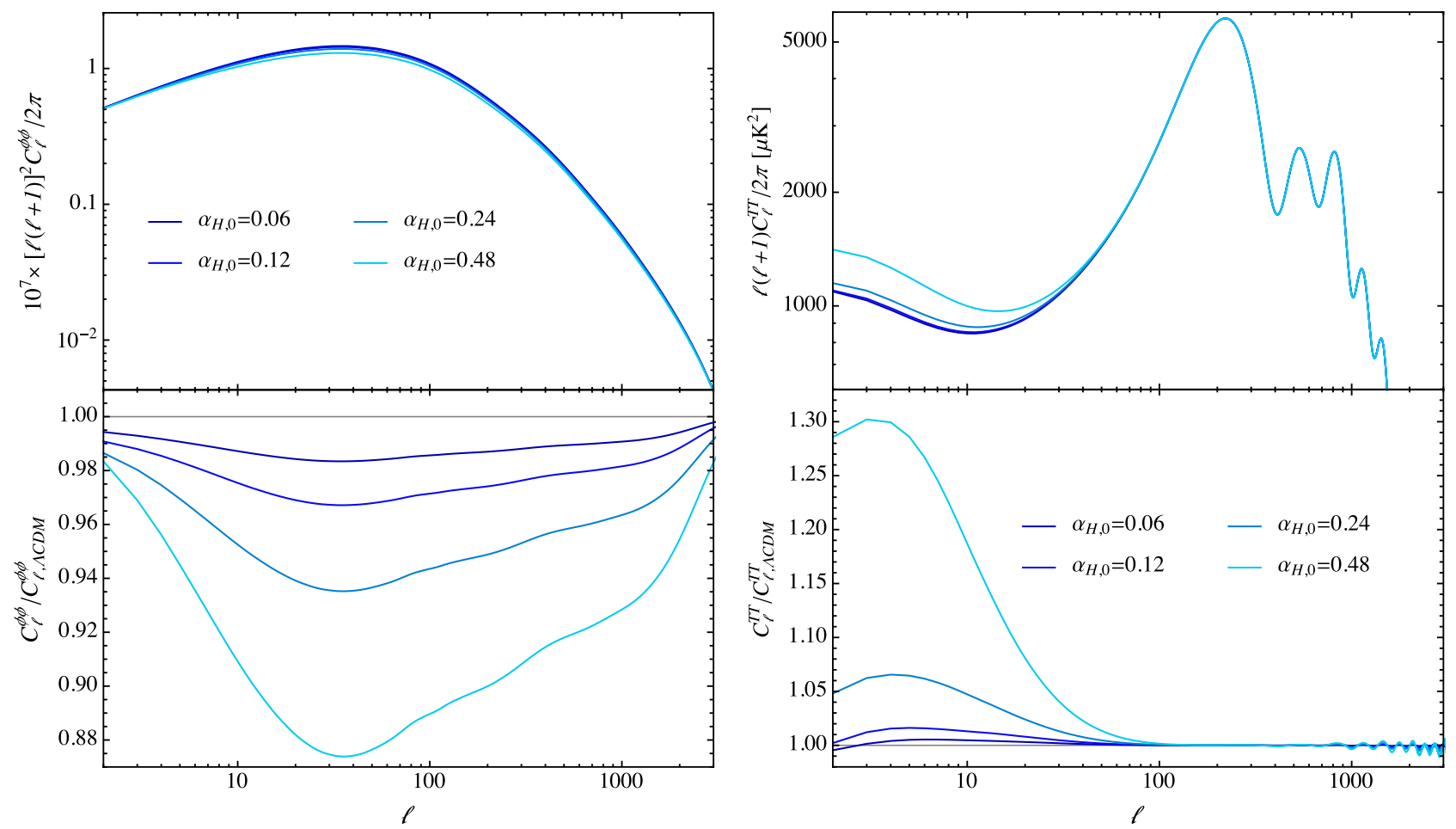

Figure 3: Effect of KMM $\left(\alpha_{\mathrm{H}}\right)$ on the CMB lensing potential (left panel) and on the CMB anisotropies (right panel) angular power spectra. The lower plots display the ratio of these angular spectra with the respective spectra for $\alpha_{\mathrm{H}}=0$.

multipole $l$. As a rough approximation, we can understand the CMB lensing potential by looking at the Weyl potential $(\Phi+\Psi) / 2$ in the quasi-static regime, i.e. using eq. (3.29). Indeed, the bulk of the CMB lensing kernel is at $0.5 \lesssim z \lesssim 6$ [76], where deviations from this approximation are below $\sim 5 \%$ for the values of $\alpha_{\mathrm{H}, 0}$ that we considered.

Let us define the quantity [50]

$$
\mu_{\mathrm{WL}} \equiv \frac{2 \nabla^{2}(\Phi+\Psi)}{3 a^{2} H^{2} \Omega_{\mathrm{m}} \delta_{\mathrm{m}}} .
$$

For $\Lambda \mathrm{CDM}, \mu_{\mathrm{WL}}=2$; in general, this quantity characterizes the deviations in weak lensing observables from the $\Lambda$ CDM case. This definition cannot be directly applied to eq. (3.29), because of the presence of the terms proportional to $\dot{\delta}_{\mathrm{m}}$ on the right-hand side of this equation. In the presence of KMM, $\alpha_{\mathrm{H}} \neq 0$, these terms equally contribute to the modifications of the Weyl potential as those proportional to $\delta_{\mathrm{m}}$ and cannot be neglected. However, a fair approximation to simplify the discussion is to replace $\dot{\delta}_{\mathrm{m}}$ by its expression in matter domination, $\dot{\delta}_{\mathrm{m}} \simeq H \delta_{\mathrm{m}}$. Setting $\alpha_{\mathrm{B}}=\alpha_{\mathrm{M}}=\alpha_{\mathrm{T}}=0$ and employing the approximation above in eq. (3.29), the effect of $\alpha_{\mathrm{H}}$ in weak lensing observables can be rewritten as

$$
\mu_{\mathrm{WL}}-2=\alpha_{\mathrm{H}} \frac{8-9 \Omega_{\mathrm{m}}\left(1+\Omega_{\mathrm{m}}\right)}{2+3\left(1-\alpha_{\mathrm{H}}\right) \Omega_{\mathrm{m}}} .
$$

One can verify that this quantity is negative for $z \gtrsim 0.5$, i.e. inside the bulk of the CMB lensing 
kernel. Therefore, the lensing potential is suppressed by the modification of gravity induced by $\alpha_{\mathrm{H}}$. For small $\Omega_{\mathrm{DE}}$, in matter domination this suppression is roughly proportional to $\alpha_{\mathrm{H}}$, as observed in Fig. 3. Expanding at linear order in $\Omega_{\mathrm{DE}}$, the above relation simplifies to $\mu_{\mathrm{WL}}-2=$ $-2 \alpha_{\mathrm{H}}+\mathcal{O}\left(\Omega_{\mathrm{DE}}^{2}\right)$.

Let us now turn to the CMB anisotropies, right panel of Fig. 3. At large $l$, the anisotropies are completely unaffected by the KMM because they are generated at recombination, ${ }^{10}$ when $\alpha_{\mathrm{H}}$ vanishes. The only visible effect is an oscillating pattern observed at high $l$ (noticeable in the lower right panel of Fig. 3.), due to the change in the CMB lensing discussed above. Indeed, lensing smears the CMB acoustic peaks; for larger values of $\alpha_{\mathrm{H}, 0}$ the smearing is suppressed and CMB peaks enhanced.

At low $l$, the deviations from the $\Lambda$ CDM case are dominated by the ISW effect, which depends on the time variation of the Weyl potential, i.e.

$$
\frac{\Delta T}{T}^{\mathrm{ISW}}(\hat{n})=-\int_{0}^{z_{*}} d z\left[\partial_{z} \Phi(\chi \hat{n}, z)+\partial_{z} \Psi(\chi \hat{n}, z)\right] .
$$

Taking the derivative of eq. (4.23) with respect to the $e$-foldings, one obtains the following relation, which only holds in the quasi-static limit:

$$
\left.\frac{d \ln (\Phi+\Psi)}{d \ln a}\right|_{\mathrm{QS}}=f_{\mathrm{QS}}-1+\frac{d \ln \mu_{\mathrm{WL}}}{d \ln a},
$$

where

$$
\left.f_{\mathrm{QS}} \equiv \frac{d \ln \delta_{\mathrm{m}}}{d \ln a}\right|_{\mathrm{QS}},
$$

is the growth rate computed using the quasi-static approximation. In $\Lambda \mathrm{CDM}, \mu_{\mathrm{WL}}=2$ and the time variation of $\Phi+\Psi$ is given by the first two terms on the right-hand side, i.e. the deviation of the matter growth rate from unity, which is negative. When gravity is modified, the last term on the right-hand side does not vanish. In the case of KMM, it contributes with the same sign as the first term, enhancing the ISW effect. For example, assuming matter domination and expanding in $\alpha_{\mathrm{H}}$ one finds

$$
\frac{d \ln \mu_{\mathrm{WL}}}{d \ln a}=-3 \alpha_{\mathrm{H}}+\mathcal{O}\left(\Omega_{\mathrm{DE}}^{2}\right)
$$

which explains the enhancement in the ISW effect observed in the right panel of Fig. (3), roughly proportional to $\alpha_{\mathrm{H}}$.

\subsection{Short-scale tension}

An intriguing issue that recently came up is the tension between the overall normalization of density fluctuations on large scales, inferred from the CMB anisotropies, and the amplitude of density fluctuations on small scales, measured with the large scale structures at low redshift. In particular, the value of $\sigma_{8}$ - defined as the rms of the fractional density fluctuation in a sphere of $8 h^{-1} \mathrm{Mpc}$ - computed from the weak lensing measurements of the Canada-France Hawaii Telescope Lensing Survey (CFHTLens) [77-80] and from cluster counts [81-83] appears to be lower than the one inferred from CMB measurements by Planck $[66,67]$. This tension has been recently confirmed by the tomographic weak gravitational lensing analysis of the Kilo Degree Survey (KiDS) [84],

\footnotetext{
${ }^{10}$ Because of this, polarization is also unaffected. For this reason we only show the temperature spectrum.
} 


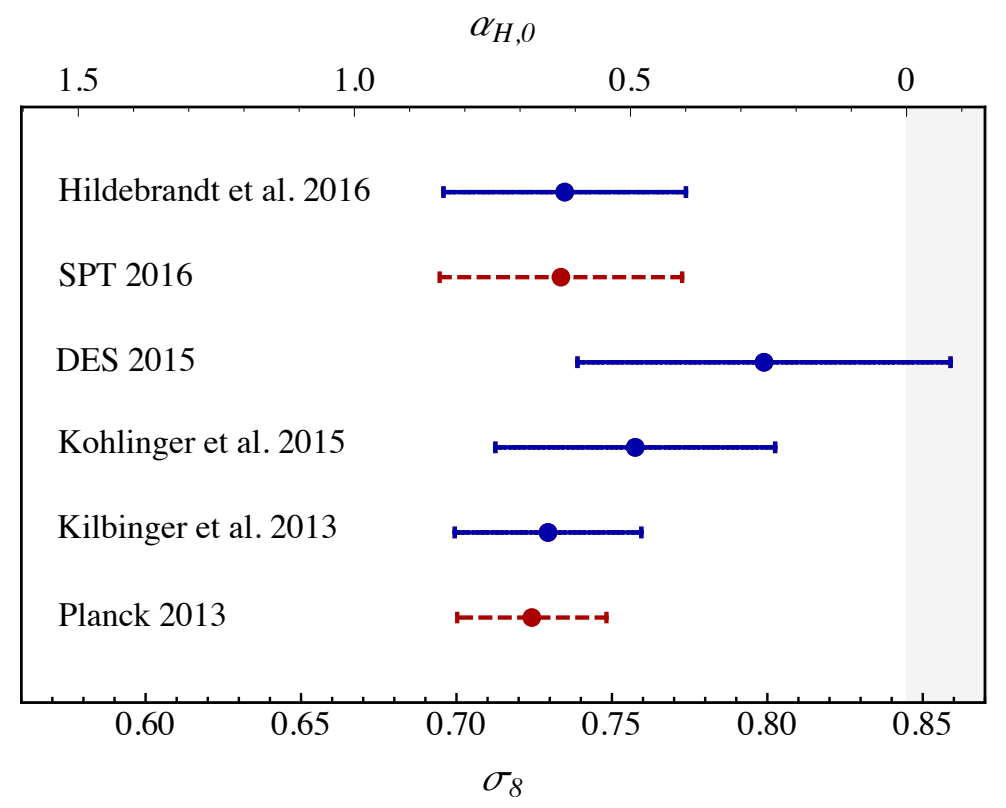

Figure 4: Relation between $\alpha_{\mathrm{H}, 0}$ and the corresponding $\sigma_{8}$ at redshift $z=0$, calculated using eq. (4.29), respectively in the top and bottom $x$-axes. The $\alpha_{\mathrm{H}, 0}=0$ line corresponds to $\Lambda \mathrm{CDM}$ and the region $\alpha_{\mathrm{H}, 0}<0$ is shaded because it is out of the stability window (4.6). The plot also shows the measurements of $\sigma_{8}$ and their respective $1-\sigma$ errors from several collaborations. ${ }^{11}$ In particular, the constraints based on cluster counts (red dashed lines) are from Planck 2013 [81] and SPT 2016 [83]. The constraints based on weak lensing observations (blue solid lines) are from several analysis of the CFHTLens, by Kilbinger et al. 2013 [77], Köhlinger et al. 2015 [80] and Hildebrandt et al. 2016 [84], and from the cosmic shear study of DES 2015 [87].

while it has been alleviated by the analysis of the latest data of the SDSS-III Baryon Oscillation Spectroscopic Survey [85]. Another aspect of this tension is reflected in redshift space distortion measurements [86], which indicate that the combination of $f \sigma_{8}$-where $f \equiv d \ln \delta_{\mathrm{m}} / d \ln a$ is the growth factor - is lower with respect to the value inferred from the Planck results.

Even though the tension is not extremely significant and depends on the uncertainties of the modeling of the non-linear scales and, for the redshift-space distortion measurements, of the galaxy bias, it might indicate a deviation from the concordance model. For instance, some attempts have been made to solve this tension using massive (active and sterile) neutrinos [89,90]. However, the most recent Planck analysis seems to disfavour this solution [67].

Given that little is known of the clustering properties of dark energy, it is natural to try to explain this tension by considering a model where deviations from the concordance one are restricted only on short scales. A recent proposal in this direction has been undertaken in [91] by exploiting the so-called "dark degeneracy" between dark matter and dark energy [92] and replacing part of the dark matter by a perfect-fluid clustering dark energy with sound speed of fluctuations smaller than unity (see for instance [93,94] for a phenomenological study of clustering dark energy

\footnotetext{
${ }^{11}$ An analysis of the effects of systematics on the CFHTLenS data, not shown in Fig. 4, has been carried out by Joudaki et al. in [88]. Moreover, we show the Planck 2013 cluster-based constraint because the more recent analysis by the Planck collaboration [82] did not release numerical values. However, the Planck 2015 results were found in agreement with the previous ones [83].
} 


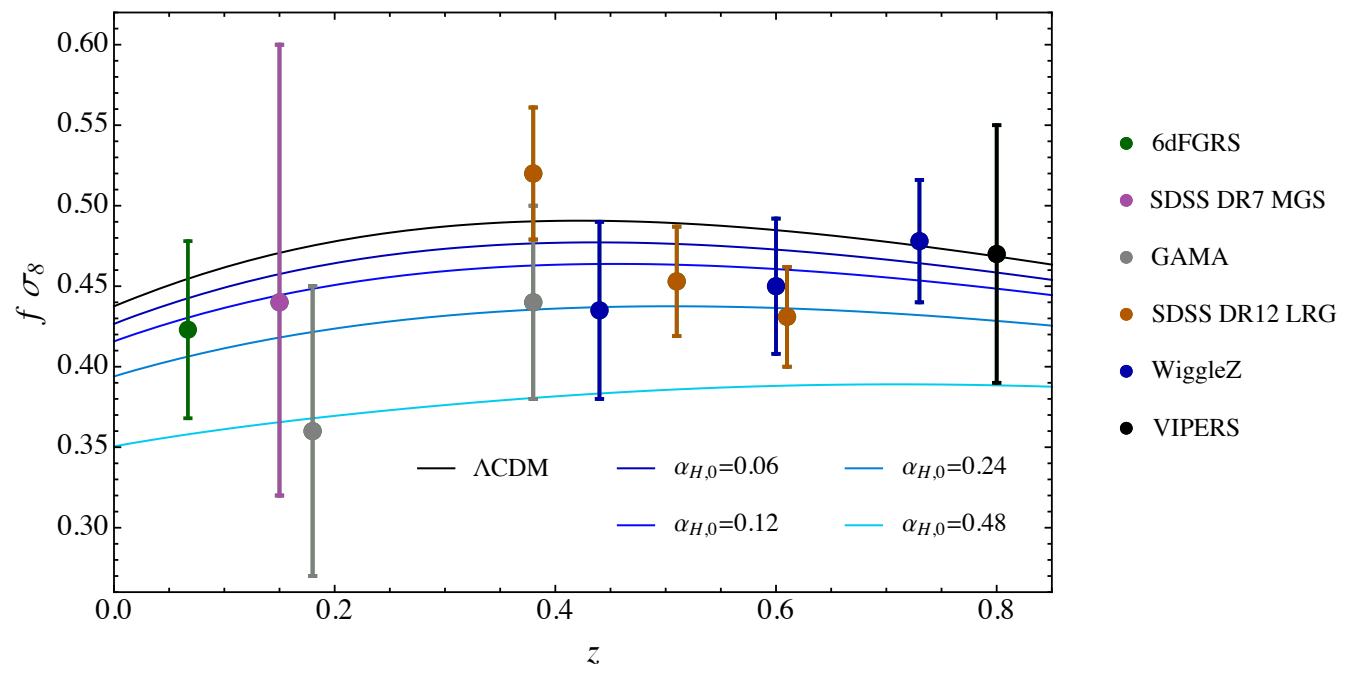

Figure 5: The quantity $f \sigma_{8}$ as a function of redshift for different values of $\alpha_{\mathrm{H}, 0}$. The plot also shows the measurements of $f \sigma_{8}$ and their respective 1- $\sigma$ errors from several redshift surveys: 6dF GRS [96], SDSS DR7 MGS [97], GAMA [98], SDSS DR12 LRG [85], WiggleZ [99] and VIPERS [100]. When possible, we plotted conditional constraints assuming a $\Lambda$ CDM background cosmology with Planck 2015 parameters. In particular, the WiggleZ constraints were taken from Fig. 16 of [67].

in the zero sound-speed limit).

More generally, one could try to leave untouched the dark matter sector and employ less specific scalar-tensor theories. For instance, it has been noted in [34] (see also [95]) that self-accelerating models within the Horndeski class with the same expansion history as $\Lambda$ CDM generally supress the linear growth rate around redshift $0.5 \lesssim z \lesssim 1$, despite the scalar fifth-force being attractive (see eq. (3.28)). Looking at eq. (3.27), this can be understood by the fact that $\Omega_{\mathrm{m}}$ on the righthand side, defined in eq. (2.14), contains the time-dependent effective Planck mass $M^{2}$ at the denominator. The enhancement of the latter due to self-acceleration lowers $\Omega_{\mathrm{m}}$ with respect to the standard $\Lambda \mathrm{CDM}$ case at intermediate redshifts, overcompensating $\mu_{\Phi}>1$.

As we have seen above, when the stability condition (4.6) is imposed the scalar force exchanged by $\pi$ in the presence of KMM is repulsive and small-scale structures are damped by a friction stronger than that provided by the Hubble expansion, see eq. (4.8), even in the absence of selfacceleration and for a $\Lambda \mathrm{CDM}$ background expansion. In light of these facts, we consider the possibility of solving the aforementioned tension with KMM.

To illustrate this, we compute $\sigma_{8}$ at redshift $z=0$ as a function of $\alpha_{\mathrm{H}, 0}$ using COOP for the cosmological parameters given at the beginning of the section. As expected from our discussion above, this yields a linear relation with $\alpha_{\mathrm{H}, 0}$, i.e.

$$
\sigma_{8} \simeq\left(0.84-0.18 \alpha_{\mathrm{H}, 0}\right) \cdot \frac{A_{s}}{2.2 \times 10^{-9}}
$$

In Fig. 4 we show this relation together with a set of large scale structure (weak lensing and cluster counts) measurements constraining $\sigma_{8}$. Two remarks are in order. First, it would be misleading to compute the value of $\alpha_{\mathrm{H}, 0}$ that best fits the data. Indeed, the constraints on $\sigma_{8}$ reported from the 
respective articles have been extracted from data assuming standard gravity. Second, as explained above large values of $\alpha_{\mathrm{H}, 0}$ yields superluminal scalar propagation. However, it is straightforward to choose a value of $\alpha_{\mathrm{K}, 0}$ such that the subluminality constraint (4.7) is satisfied, without affecting the redshift-survey scale evolution (as long as $c_{s}>0.1$, see footnote 1 ).

Moreover, to illustrate the effect of KMM on the growth rate, in Fig. 5 we plot the combination $f \sigma_{8}$ as a function of redshift for different values of $\alpha_{\mathrm{H}, 0}$. Although in the presence of KMM the growth rate $f$ is scale dependent, we can confidently use its scale-independent value computed in the quasi-static regime, $f_{\mathrm{QS}}$, see eq. (4.27), because this approximation holds on redshift-survey scales. As discussed above, we do not try to consistently fit the value of $\alpha_{\mathrm{H}, 0}$ to these observations but we note that $\alpha_{\mathrm{H}, 0} \sim$ few $\times 0.1$ would provide the hinted small-scale suppression. A too large value of $\alpha_{\mathrm{H}, 0}$ may give an unreasonably large ISW effect, see Fig. 3. However, this could be compensated by a small change in another parameter, such as the dark energy equation of state. We postpone for a future publication a more consistent analysis of the CMB and large scale structure measurements that takes into account the effects of modified gravity on the observables.

\section{$5 \quad$ Summary and conclusions}

Using the framework of the Effective Theory of Dark Energy, in this paper we studied the observational effects of Kinetic Matter Mixing, i.e. a kinetic coupling between matter and the cosmological scalar field, which is present if matter is disformally coupled to the gravitational sector with a disformal coupling that depends on the first derivative of the scalar field or in theories beyond Horndeski.

In Sec. 2, we started by discussing the most generic quadratic action for cosmological perturbations in the presence of conformal and disformal couplings of matter to the gravitational sector, under the assumption that the disformal factor depends as well on the first derivative of the scalar field, other than its value. Moreover, we showed that a change of frame does not change the structure of the action but redefines the coefficients of the various operators. In particular, the coefficient of the operator that characterizes theories beyond the Horndeski class is redefined only by the dependence of the disformal coupling on the field derivative. This is explicitly shown by the frame-independent parameter $\lambda^{2}$, defined in eq. (2.17), which measures the degree of Kinetic Matter Mixing. By diagonalizing the kinetic action, we derived the conditions that one must require for the perturbations to be free of ghosts and of gradient instabilities (the generalization to multiple matter species is given in App. A).

After this general frame-independent description, in Sec. 3 we assumed that matter is universally coupled and, without loss of generality, we considered the case where it is also minimally coupled, i.e. the Jordan frame description, where observational predictions are more easily derived. We then discussed the short-scale regime and derived the eigenmodes of the acoustic oscillations, which are mixed states of matter and the scalar field waves. Focussing on the case where matter is made of nonrelativistic particles (such as cold dark matter or baryons) we derived the equations in the quasi-static approximation and discussed (see App. C) how the quasi-static regime is reached during the cosmological evolution. These equations allow for a clear analytical understanding of the effects of modifications of gravity due to Kinetic Matter Mixing. In particular, while models in the Horndeski class only modify the Poisson equation with an effective Newton constant, Kinetic Matter Mixing also induces an additional friction term. Remarkably, requiring the stability conditions implies that gravity is weakened on short scales, an effect which is hard to reproduce 
in models within the Horndeski class. Finally, by comparing the quasi-static solution to the full numerical one, we showed that the quasi-static limit approximates very well the dynamics on scales shorter than the sound horizon.

In Sec. 4 we focussed on the cosmological effects of the beyond Horndeski operator, obtaining the full numerical solutions using the publicly available Einstein-Boltzmann solver of COOP [47]. Using these solutions, we derived the matter power spectrum at two different redshifts, and the angular spectra of the CMB lensing potential and of the CMB anisotropies. On small scales, i.e. for $k \gtrsim$ few $\times 10^{-3} \mathrm{Mpc}$, the solution matches the quasi-static regime and the matter power spectrum is suppressed independently of $k$. An analytical study of the large scales is complicated by the complexity of the full system of equations. However, we obtained analytical solutions on these scales by perturbing around the $\Lambda \mathrm{CDM}$ solutions for small Kinetic Matter Mixing. The agreement with the numerical solution is excellent. Moreover, its simplicity allows an immediate understanding of the behavior of the perturbations and their observables. Similarly to the matter power spectrum, also the angular spectrum of the CMB lensing potential is suppressed. The CMB anisotropy is affected at very low multipoles through the ISW effect, which is enhanced, and on very high multipoles because of the suppression of the lensing potential.

In App. D, we compared this case with the one of kinetic braiding, which displays qualitatively opposite effects. Also in this case we studied analytically the large-scale behavior and derived the value of the crossing scale, i.e. the scale at which the power spectrum displays the transition between the short-scale enhancement and large-scale suppression.

As mentioned above, Kinetic Matter Mixing appears as the only modification of gravity in the context of single-field models that weakens the strength of gravity on small scales. Therefore, in Sec. 4.3 we entertained the possibility that the tension between the Planck data and smallscale observations can be explained by this effect. In particular, as shown in Figs. 4 and 5, KMM predicts a lower value of $\sigma_{8}$ and $f \sigma_{8}$, which could be made compatible with those measured by weak lensing and redshift-space distortion observations. We postpone to future work a more consistent dedicated analysis that marginalizes over the other cosmological parameters.

In summary, we presented a robust theoretical understanding of the effects of Kinetic Matter Mixing across different observables and scales. These effects may be a smoking gun of modified gravity for the next observational missions and a complete forecast, taking into account the characteristics of the next missions, is an obvious next step.

Acknowledgements: It is a pleasure to thank Jérôme Gleyzes, Martin Kunz, David Langlois, Jean-Baptiste Melin, Federico Piazza, Ignacy Sawicki and Jeremy Tinker for useful discussions. M.M. and F.V. also thank the Theoretical Physics Departments of CERN and Université de Genève for hospitality during large part of this work. G. D'A. thanks the CCPP at New York University for hospitality during the final stages of this work.

\section{A Quadratic action and stability for multiple species}

Here we study the linear stability of the gravitational and matter action, extracting the propagating degrees of freedom and their speed of propagation. To this end, we will expand the total action up to quadratic order in linear scalar fluctuations around a FLRW solution and solve the constraints, generalizing the treatment of [50] by including $\alpha_{\mathrm{H}}$ and the dependence on $X$ of the disformal 
functions $D_{I}$. When not explicitly given, the details of the calculation can be found in this reference.

To describe the matter sector, we extend the treatment of the main text and assume that the universe is filled by $N_{S}$ matter species labelled by an index $I$, with $I=1, \ldots, N_{S}$, each minimally coupled to a different metric. For each species $I$, we denote the corresponding metric by $\check{g}_{\mu \nu}^{(I)}$ and we call this the Jordan frame metric associated with this species. The total matter action is thus given by

$$
S_{\mathrm{m}}=\sum_{I}^{N_{S}} S_{I}, \quad S_{I}=\int d^{4} x \sqrt{-\check{g}^{(I)}} L_{I}\left(\check{g}_{\mu \nu}^{(I)}, \psi_{I}\right)
$$

with

$$
\check{g}_{\mu \nu}^{(I)}=C_{I}^{(\phi)}(\phi) g_{\mu \nu}+D_{I}^{(\phi)}(\phi, X) \partial_{\mu} \phi \partial_{\nu} \phi
$$

$\left(C_{I}^{(\phi)}>0\right.$ in order to preserve the Lorentzian signature of the Jordan-frame metric of the species I.) As usual, one can use the arbitrariness in the choice of the gravitational metric $g_{\mu \nu}$ to choose one particular matter species, say $I_{*}$, to be minimally coupled to it, in which case we have $C_{I_{*}}^{(\phi)}=1$ and $D_{I_{*}}^{(\phi)}=0$. This defines its Jordan metric as the gravitational metric.

It is convenient to introduce the parameters

$$
\alpha_{\mathrm{C}, I} \equiv \frac{\sqrt{-X}}{2 H} \frac{d \ln C_{I}^{(\phi)}}{d \phi}, \quad \alpha_{\mathrm{D}, I} \equiv-\frac{X D_{I}^{(\phi)}}{C_{I}^{(\phi)}+X D_{I}^{(\phi)}}, \quad \alpha_{\mathrm{X}, I} \equiv \frac{X^{2}}{C_{I}^{(\phi)}} \frac{\partial D_{I}^{(\phi)}}{\partial X}
$$

where the right-hand side is evaluated on the background. (Requiring a Lorentzian Jordan frame metric implies $\alpha_{\mathrm{D}, I}>-1$ [51].) In unitary gauge, eq. (A.2) reads

$$
\check{g}_{\mu \nu}^{(I)}=C_{I}(t) g_{\mu \nu}+D_{I}(t, N) \delta_{\mu}^{0} \delta_{\nu}^{0},
$$

with

$$
C_{I}(t)=C_{I}^{(\phi)}(\phi(t)), \quad D_{I}(t, N)=\dot{\phi}^{2}(t) D_{I}^{(\phi)}\left(\phi(t),-\dot{\phi}(t)^{2} / N^{2}\right) .
$$

Then, the above parameters read

$$
\alpha_{\mathrm{C}, I}=\frac{\dot{C}_{I}}{2 H C_{I}}, \quad \alpha_{\mathrm{D}, I}=\frac{D_{I}}{C_{I}-D_{I}}, \quad \alpha_{\mathrm{X}, I}=-\frac{1}{2 C_{I}} \frac{\partial D_{I}}{\partial N}
$$

Let us start by expanding the matter action. For simplicity, we assume that each matter species can be described by a perfect fluid with vanishing vorticity. It is then easy to write an action in terms of derivatively coupled scalar fields with Lagrangians of the form [101-103]

$$
L_{I}\left(\check{g}_{\mu \nu}^{(I)}, \psi_{I}\right) \equiv P_{I}\left(Y_{I}\right), \quad Y_{I} \equiv \check{g}_{(I)}^{\mu \nu} \partial_{\mu} \sigma_{I} \partial_{\nu} \sigma_{I}
$$

Splitting each scalar field $\sigma_{I}$ into a background value and its perturbations, $\sigma_{I}=\bar{\sigma}_{I}(t)+\delta \sigma_{I}(t, \mathbf{x})$, the second-order expansion $S_{I}$ reads

$$
\begin{aligned}
S_{I}^{(2)}= & \int d^{3} x d t \frac{a^{3} \rho_{I}}{c_{s, I}^{2}}\left\{g_{\delta N^{2}, I} \delta N^{2}+\frac{1+\left(1+\alpha_{\mathrm{D}, I}\right) w_{I}}{\left(1+\alpha_{\mathrm{D}, I}\right)^{2}} \frac{1}{2 \dot{\bar{\sigma}}_{I}^{2}}\left[\delta \dot{\sigma}_{I}^{2}-c_{s, I}^{2} \frac{\left(\partial_{i} \delta \sigma\right)^{2}}{a^{2}}\right]\right. \\
& \left.-\frac{1+\left(1+\alpha_{\mathrm{D}, I}\right) w_{I}}{\left(1+\alpha_{\mathrm{D}, I}\right)^{2}} \frac{1}{\dot{\bar{\sigma}}_{I}}\left[\delta \dot{\sigma}_{I}\left(\delta N-c_{s, I}^{2} \delta \sqrt{h}\right)+c_{s, I}^{2} N^{i} \partial_{i} \delta \sigma_{I}\right]\right\},
\end{aligned}
$$


where we have defined

$$
g_{\delta N^{2}, I} \equiv c_{s, I}^{2} \alpha_{\mathrm{D}, I}^{\mathrm{eff}}+\left(1+\alpha_{\mathrm{X}, I}\right)^{2}\left[1+w_{I}\left(1+\alpha_{\mathrm{D}, I}\right)\right]
$$

with the combination

$$
\alpha_{\mathrm{D}, I}^{\mathrm{eff}} \equiv \alpha_{\mathrm{D}, I}\left(1+\alpha_{\mathrm{X}, I}\right)^{2}+\alpha_{\mathrm{X}, I}\left(2+\alpha_{\mathrm{X}, I}\right)+\frac{1}{2 C_{I}} \frac{\partial^{2} D_{I}}{\partial N^{2}}
$$

and the fluid quantities

$$
\begin{aligned}
p_{I} & \equiv \frac{C_{I}^{2}}{\sqrt{1+\alpha_{\mathrm{D}, I}}} P_{I}, \quad \rho_{I} \equiv C_{I}^{2} \sqrt{1+\alpha_{\mathrm{D}, I}}\left(2 Y_{I} P_{I}^{\prime}-P_{I}\right) \\
c_{s, I}^{2} & \equiv \frac{P_{I}^{\prime}}{P_{I}^{\prime}+2 Y_{I} P_{I}^{\prime \prime}}\left(1+\alpha_{\mathrm{D}, I}\right)^{-1} .
\end{aligned}
$$

Here a prime denotes a derivative with respect to the variable $Y_{I}$. We have omitted in the action irrelevant terms that vanish when imposing the background equations of motion.

We can now investigate the stability of scalar perturbations. The full second-order action

$$
S^{(2)}=S_{\mathrm{g}}^{(2)}+S_{\mathrm{m}}^{(2)}
$$

where the gravitational part $S_{\mathrm{g}}^{(2)}$ is given in eq. (2.5), governs the dynamics of linear scalar fluctuations. The scalar modes can be described in unitary gauge by defining $\psi \equiv \partial^{-2} \partial_{i} N^{i}$ and writing the spatial metric as $h_{i j}=a^{2}(t) e^{2 \zeta} \delta_{i j}$ [104]. Variation with respect to $\psi$ yields the (scalar part of) the momentum constraint, and its solution can be used to replace $\delta N$ in terms of $\dot{\zeta}$ and $\delta \sigma_{I}$ into the second-order action (see details in Ref. [50]). Re-expressing the scalar field perturbations $\delta \sigma_{I}$ in terms of the gauge invariant variables $\mathcal{Q}_{I} \equiv \delta \sigma-\left(\dot{\bar{\sigma}}_{I} / H\right) \zeta$, the total second-order action reads, focusing only on the kinetic and spatial gradient parts,

$$
\begin{aligned}
S^{(2)}= & \int d^{3} x d t a^{3} \frac{M^{2}}{2}\left[g_{\dot{\zeta} \dot{\zeta}} \dot{\zeta}^{2}-g_{\partial \zeta \partial \zeta} \frac{\left(\partial_{i} \zeta\right)^{2}}{a^{2}}+\sum_{I} \frac{\kappa_{I} H^{2}}{\dot{\bar{\sigma}}_{I}^{2} c_{s, I}^{2}}\left(\dot{\mathcal{Q}}_{I}^{2}-c_{s, I}^{2} \frac{\left(\partial_{i} \mathcal{Q}_{I}\right)^{2}}{a^{2}}\right)\right. \\
& \left.+2 \sum_{I} \frac{H}{c_{s, I}^{2} \dot{\bar{\sigma}}_{I}}\left(g_{\dot{\mathcal{Q}} \dot{\zeta}, I} \dot{\mathcal{Q}}_{I} \dot{\zeta}-g_{\partial \mathcal{Q} \partial \zeta, I} \frac{c_{s, I}^{2}}{a^{2}} \partial_{i} \mathcal{Q}_{I} \partial_{i} \zeta\right)\right],
\end{aligned}
$$

with

$$
\begin{aligned}
g_{\dot{\zeta} \dot{\zeta}} & \equiv \frac{1}{\left(1+\alpha_{\mathrm{B}}\right)^{2}}\left\{\alpha+\sum_{I} \frac{\kappa_{I}}{c_{s, I}^{2}}\left[1+\alpha_{\mathrm{B}}-\left(1+\alpha_{\mathrm{X}, I}\right)\left(1+\alpha_{\mathrm{D}, I}\right)\right]^{2}\right\}, \\
g_{\partial \zeta \partial \zeta} & \equiv \frac{c_{s, 0}^{2}\left(\alpha_{\mathrm{K}}+6 \alpha_{\mathrm{B}}^{2}\right)}{\left(1+\alpha_{\mathrm{B}}\right)^{2}}+\sum_{I} \frac{\kappa_{I}}{1+\alpha_{\mathrm{B}}}\left[1+\alpha_{\mathrm{B}}-2\left(1+\alpha_{\mathrm{H}}\right)\left(1+\alpha_{\mathrm{D}, I}\right)\right], \\
g_{\dot{\mathcal{Q}} \dot{\zeta}, I} & \equiv \frac{\kappa_{I}}{1+\alpha_{\mathrm{B}}}\left[1+\alpha_{\mathrm{B}}-\left(1+\alpha_{\mathrm{X}, I}\right)\left(1+\alpha_{\mathrm{D}, I}\right)\right], \\
g_{\partial \mathcal{Q} \partial \zeta, I} & \equiv \frac{\kappa_{I}}{1+\alpha_{\mathrm{B}}}\left[1+\alpha_{\mathrm{B}}-\left(1+\alpha_{\mathrm{H}}\right)\left(1+\alpha_{\mathrm{D}, I}\right)\right],
\end{aligned}
$$


where we defined the dimensionless coefficient

$$
\begin{aligned}
\alpha & \equiv \alpha_{\mathrm{K}}+6 \alpha_{\mathrm{B}}^{2}+3 \sum_{I} \alpha_{\mathrm{D}, I}^{\mathrm{eff}} \Omega_{I} \geq 0 \\
\kappa_{I} & \equiv 3 \frac{1+\left(1+\alpha_{\mathrm{D}, I}\right) w_{I}}{\left(1+\alpha_{\mathrm{D}, I}\right)^{2}} \Omega_{I}, \\
c_{s, 0}^{2} & \equiv \frac{\left(1+\alpha_{\mathrm{B}}\right)^{2}}{\alpha_{\mathrm{K}}+6 \alpha_{\mathrm{B}}^{2}}\left\{2\left(1+\alpha_{\mathrm{T}}\right)-\frac{2}{a M^{2}} \frac{d}{d t}\left[\frac{a M^{2}\left(1+\alpha_{\mathrm{H}}\right)}{H\left(1+\alpha_{\mathrm{B}}\right)}\right]\right\} .
\end{aligned}
$$

Absence of ghosts is ensured by requiring that the matrix of the kinetic coefficients is positive definite, which yields the conditions $\alpha \geq 0$ and $\kappa_{I} \geq 0$. The second condition reads $\rho_{I}+(1+$ $\left.\alpha_{\mathrm{D}, I}\right) p_{I} \geq 0$, which is the usual Null Energy Condition written in a disformally related frame.

Requiring that the determinant of the kinetic matrix vanishes yields the dispersion relation

$$
\left(\omega^{2}-c_{s}^{2} k^{2}\right) \prod_{I}^{N_{S}}\left(\omega^{2}-c_{s, I}^{2} k^{2}\right)=\frac{3}{\alpha} \omega^{2} k^{2} \sum_{I}\left[1+\left(1+\alpha_{\mathrm{D}, I}\right) w_{I}\right] \Omega_{I}\left(\alpha_{\mathrm{H}}-\alpha_{\mathrm{X}, I}\right)^{2} \prod_{J \neq I}^{N_{S}}\left(\omega^{2}-c_{s, J}^{2} k^{2}\right),
$$

where the scalar sound speed squared $c_{s}^{2}$ is given by

$$
c_{s}^{2} \equiv c_{s, 0}^{2} \frac{\alpha_{\mathrm{K}}+6 \alpha_{\mathrm{B}}^{2}}{\alpha}-\frac{\left(1+\alpha_{\mathrm{H}}\right)^{2}}{\alpha} \sum_{I} \kappa_{I}\left(1+\alpha_{\mathrm{D}, I}\right)^{2} .
$$

For a single matter fluid this yields eq. (2.16). In the absence of a disformal coupling, $\alpha_{\mathrm{D}, I}=$ $\alpha_{\mathrm{X}, I}=0$, we recover the results of $[27,28]$. If the disformal coupling does not depend on $X$, $\alpha_{\mathrm{X}, I}=0$, and we restrict to Horndeski theories, $\alpha_{\mathrm{H}}=0$, we recover the results of [50].

\section{B Quadratic action in Newtonian gauge}

The second-order action (2.5) can be written in Newtonian gauge, eq. (3.1), after a time diffeomorphism $t \rightarrow t+\pi(t, \vec{x})$. This reads

$$
\begin{aligned}
S_{\text {grav }}^{(2)}= & \int d^{4} x a^{3} M^{2}\left\{\frac{1}{2} H^{2} \alpha_{\mathrm{K}} \dot{\pi}^{2}+\left[\dot{H}+\frac{1}{2 M^{2}}\left(\rho_{\mathrm{m}}+p_{\mathrm{m}}+2\left(M^{2} H\left(\alpha_{\mathrm{B}}-\alpha_{\mathrm{H}}\right)\right)^{\cdot}\right)\right.\right. \\
& \left.+H^{2}\left(\alpha_{\mathrm{B}}-\alpha_{\mathrm{M}}+\alpha_{\mathrm{T}}-\alpha_{\mathrm{H}}\right)\right] \frac{(\nabla \pi)^{2}}{a^{2}}-3 \dot{\Psi}^{2}+\left(1+\alpha_{\mathrm{T}}\right) \frac{(\nabla \Psi)^{2}}{a^{2}}-2\left(1+\alpha_{\mathrm{H}}\right) \frac{\nabla \Phi \nabla \Psi}{a^{2}} \\
& +2 \alpha_{\mathrm{H}} \frac{\nabla \dot{\pi} \nabla \Psi}{a^{2}}+2 H\left(\alpha_{\mathrm{B}}-\alpha_{\mathrm{H}}\right) \nabla \Phi \nabla \pi-2 H\left(\alpha_{\mathrm{M}}-\alpha_{\mathrm{T}}\right) \frac{\nabla \Psi \nabla \pi}{a^{2}}+6 H \alpha_{\mathrm{B}} \dot{\pi} \dot{\Psi} \\
& +H^{2}\left(6 \alpha_{\mathrm{B}}-\alpha_{\mathrm{K}}\right) \Phi \dot{\pi}-6 H\left(1+\alpha_{\mathrm{B}}\right) \dot{\Psi} \Phi-6\left(\frac{\rho_{\mathrm{m}}+p_{\mathrm{m}}}{2 M^{2}}+\dot{H}\right) \dot{\Psi} \pi \\
& +\left[H^{2}\left(\frac{1}{2} \alpha_{\mathrm{K}}-3\left(1+2 \alpha_{\mathrm{B}}\right)\right)+\frac{\rho_{\mathrm{m}}}{2 M^{2}}\right] \Phi^{2}-\frac{9 p_{\mathrm{m}}}{2 M^{2}} \Psi^{2}-\frac{3 \rho_{\mathrm{m}}}{M^{2}} \Phi \Psi \\
& -3 H\left(\frac{\rho_{\mathrm{m}}+p_{\mathrm{m}}}{2 M^{2}}+\dot{H}\left(1+\alpha_{\mathrm{B}}\right)\right) \Phi \pi \\
& \left.-3\left[\dot{H}\left(\frac{\rho_{\mathrm{m}}+p_{\mathrm{m}}}{2 M^{2}}+\dot{H}\right)+H\left(\alpha_{\mathrm{B}} \dot{H}\right)^{\cdot}+H^{2} \dot{H} \alpha_{\mathrm{B}}\left(3+\alpha_{\mathrm{M}}\right)+\alpha_{\mathrm{B}} \dot{H}^{2}\right] \pi^{2}\right\} .
\end{aligned}
$$


In Newtonian gauge, the matter action (3.2) expanded at second-order reads

$$
\begin{aligned}
S_{\mathrm{m}}^{(2)}= & \int d^{4} x a^{3}\left\{\frac{9}{2} p_{\mathrm{m}} \Psi^{2}+\frac{1}{2}\left[\frac{p_{\mathrm{m}}+\left(1-c_{\mathrm{m}}^{2}\right) \rho_{\mathrm{m}}}{c_{\mathrm{m}}^{2}}\right] \Phi^{2}+3 \rho_{\mathrm{m}} \Phi \Psi\right. \\
& \left.+\frac{\rho_{\mathrm{m}}+p_{\mathrm{m}}}{2 c_{\mathrm{m}}^{2} \dot{\sigma}_{0}^{2}}\left[\dot{\delta \sigma}^{2}-c_{\mathrm{m}}^{2} \frac{(\nabla \delta \sigma)^{2}}{a^{2}}\right]-\frac{\rho_{\mathrm{m}}+p_{\mathrm{m}}}{c_{\mathrm{m}}^{2} \dot{\sigma}_{0}}\left(\Phi+3 c_{\mathrm{m}}^{2} \Psi\right) \dot{\delta \sigma}\right\} .
\end{aligned}
$$

\section{From the oscillating to the quasi-static regime}

We can discuss the transition from the oscillating to the quasi-static regime starting from the linearized equations of motion for $\Psi$ in the presence of matter, once the $\pi$ field has been integrated out $[10,26,33]$. On short scales, in Fourier space the variation of the action with respect to $\Psi$ and the relation $\Phi_{E}=\Psi_{E}$ read, respectively,

$$
\begin{aligned}
\ddot{\Psi}+\left(3+b_{1}\right) H \dot{\Psi}+c_{s}^{2} \frac{k^{2}}{a^{2}} \Psi & =-\frac{3}{2} \Omega_{\mathrm{m}} H^{2}\left[c_{s}^{2} \mu_{\Psi} \delta_{\mathrm{m}}-\frac{2 \alpha_{\mathrm{H}}\left(\alpha_{\mathrm{B}}-\alpha_{\mathrm{H}}\right)}{\alpha} \frac{k^{2}}{a^{2} H^{2}} H v_{\mathrm{m}}\right], \\
\frac{\alpha_{\mathrm{H}}}{\alpha_{\mathrm{B}}-\alpha_{\mathrm{H}}} \frac{\dot{\Psi}}{H}+b_{2} \Psi-\Phi & =-\frac{3}{2} \Omega_{\mathrm{m}} \frac{a^{2} H^{2}}{k^{2}} b_{3} \delta_{\mathrm{m}}+\frac{3}{2} \frac{\alpha_{\mathrm{H}}}{\alpha_{\mathrm{B}}-\alpha_{\mathrm{H}}} \Omega_{\mathrm{m}} H v_{\mathrm{m}},
\end{aligned}
$$

where the specific form of the coefficients $b_{1}, b_{2}$ and $b_{3}$ are explicitly given by

$$
\begin{aligned}
b_{1} & \equiv 3+\alpha_{\mathrm{M}}+\frac{\alpha_{\mathrm{B}}^{2}}{H \alpha}\left(\frac{\alpha_{\mathrm{K}}}{\alpha_{\mathrm{B}}^{2}}\right)^{\cdot}+\alpha_{\mathrm{H}}\left[\frac{2}{\alpha_{\mathrm{H}}-\alpha_{\mathrm{B}}}\left(\frac{\dot{\alpha}_{\mathrm{B}}}{H \alpha_{\mathrm{B}}}-\frac{\dot{\alpha}_{\mathrm{H}}}{H \alpha_{\mathrm{H}}}\right)+\frac{3}{4} \Omega_{\mathrm{m}}\right], \\
b_{2} & \equiv \frac{1}{\left(\alpha_{\mathrm{B}}-\alpha_{\mathrm{H}}\right)^{2}}\left[\alpha_{\mathrm{B}} \xi+\alpha_{\mathrm{H}}\left(1+\alpha_{\mathrm{H}}\right)\left(\frac{\dot{H}}{H^{2}}+\frac{3}{2} \Omega_{\mathrm{m}}\right)\right], \\
b_{3} & \equiv \frac{1}{\left(\alpha_{\mathrm{B}}-\alpha_{\mathrm{H}}\right)^{2}}\left[\alpha_{\mathrm{B}}\left(\alpha_{\mathrm{T}}-\alpha_{\mathrm{M}}\right)+\alpha_{\mathrm{H}}\left(\frac{\dot{H}}{H^{2}}+\frac{3}{2} \Omega_{\mathrm{m}}\right)\right] .
\end{aligned}
$$

In the standard quintessence case, for $\alpha_{\mathrm{H}}=\alpha_{\mathrm{T}}=\alpha_{\mathrm{M}}=\alpha_{\mathrm{B}}=0$, we have $b_{1}=0, b_{2}=1$ and $b_{3}=0$. Matter is described by the usual continuity and Euler equations, eqs. (3.18) and (3.26).

One cannot find an analytical solution to eqs. (C.1) and (C.2) but we can assume that the full solution can be separated into an oscillating part, with characteristic frequency $\omega \sim c_{+} k / a=$ $c_{s} \sqrt{1+\lambda^{2}} k / a$, and a "quasi-static" part, slowly evolving at a rate given by $\sim H$ [52]. For instance, for $\Psi$ one can write

$$
\Psi=\Psi_{\mathrm{osc}}+\Psi_{\mathrm{QS}}
$$

We also assume that $\Psi_{\text {osc }}$ has a slowly decaying envelop due to the expansion of the universe, such that

$$
\dot{\Psi}=\dot{f} \Psi_{\mathrm{osc}}+i \omega \Psi_{\mathrm{osc}}+\dot{\Psi}_{\mathrm{QS}}, \quad \dot{f} \sim \mathcal{O}(H), \quad\left(\ln \Psi_{\mathrm{QS}}\right)^{\cdot} \sim \mathcal{O}(H)
$$

To count the importance of each term in the above equations, we consider the limit $\omega \gg H$ and we define the following two expansion parameters,

$$
\epsilon_{k} \equiv \frac{a H}{k} \ll 1, \quad \epsilon_{\omega} \equiv \frac{H}{\omega}=\frac{a H}{c_{+} k} \ll 1
$$


Starting by defining $\Psi \sim \mathcal{O}(1)$, and using eq. (C.1) and the continuity and Euler equations, one can find

$$
\begin{aligned}
& H v_{\mathrm{QS}} \sim \mathcal{O}(1), \quad \Phi_{\mathrm{QS}} \sim \mathcal{O}(1), \quad \delta_{\mathrm{QS}} \sim \mathcal{O}\left(\epsilon_{k}^{-2}\right), \\
& H v_{\text {osc }} \sim \mathcal{O}(1), \quad \Phi_{\text {osc }} \sim \mathcal{O}\left(\epsilon_{\omega}^{-1}\right), \quad \delta_{\text {osc }} \sim \mathcal{O}\left(\epsilon_{k}^{-2} \epsilon_{\omega}\right) .
\end{aligned}
$$

At this point, we can expand the above equations in these expansion parameters. At the lowest order in $\epsilon_{k}$, the quasi-static solutions satisfy the relations discussed in Sec. 3.2, not surprisingly. For the oscillating piece, eqs. (C.1) and (C.2) become, retaining only the lowest order in $\epsilon_{\omega}$ and $\epsilon_{k}$,

$$
\begin{aligned}
-\omega^{2} \Psi_{\mathrm{osc}}+c_{s}^{2} \frac{k^{2}}{a^{2}} \Psi_{\mathrm{osc}} & \simeq 3 \Omega_{\mathrm{m}} \frac{\alpha_{\mathrm{H}}\left(\alpha_{\mathrm{B}}-\alpha_{\mathrm{H}}\right)}{\alpha} \frac{k^{2}}{a^{2}} H v_{\mathrm{osc}}, \\
\frac{\alpha_{\mathrm{H}}}{\alpha_{\mathrm{B}}-\alpha_{\mathrm{H}}} i \frac{\omega}{H} \Psi_{\mathrm{osc}}-\Phi_{\mathrm{osc}} & \simeq 0,
\end{aligned}
$$

where we have used $\dot{f} / H \simeq-\left(3+b_{1}\right)+\mathcal{O}\left(\epsilon_{\omega}\right)$. For $b_{1}>-3$, this implies that the oscillating part decays in time. For instance, for a constant $b_{1}$ the oscillating solution decays as $a^{-\left(3+b_{1}\right)}$. Combining the Euler equation $i \omega v_{\mathrm{osc}}=-\Phi_{\mathrm{osc}}$ with eq. (C.11) we get a simple relation between the velocity and the curvature, i.e. $H v_{\text {osc }} \simeq-\alpha_{\mathrm{H}} /\left(\alpha_{\mathrm{B}}-\alpha_{\mathrm{H}}\right) \Psi_{\mathrm{osc}}$. Replacing this expression in the first equation, we find the expected dispersion relation for the oscillating normal mode,

$$
\omega^{2}=c_{s}^{2} \frac{k^{2}}{a^{2}}+3 \Omega_{\mathrm{m}} \frac{\alpha_{\mathrm{H}}^{2}}{\alpha} \frac{k^{2}}{a^{2}}=c_{+}^{2} \frac{k^{2}}{a^{2}} .
$$

\section{Observational signatures of Kinetic Braiding}

It is interesting to compare the results of Sec. 4 with the case of kinetic braiding. Indeed, this modification of gravity is expected to lead to similar effects as KMM on the power spectrum and CMB anisotropies. We assume the same background expansion history as in eq. (4.4) and set, this time,

$$
\alpha_{\mathrm{H}}=\alpha_{\mathrm{M}}=\alpha_{\mathrm{T}}=0 .
$$

Moreover, we parametrize the time dependence of $\alpha_{\mathrm{K}}$ and $\alpha_{\mathrm{B}}$ as

$$
\alpha_{\mathrm{K}}=\alpha_{\mathrm{K}, 0} \frac{\Omega_{\mathrm{DE}}(t)}{\Omega_{\mathrm{DE}, 0}}, \quad \alpha_{\mathrm{B}}=\alpha_{\mathrm{B}, 0} \frac{\Omega_{\mathrm{DE}}(t)}{\Omega_{\mathrm{DE}, 0}} .
$$

Recently, in Ref. [45] an analogous parametrization has been used to discuss the effect of $\alpha_{\mathrm{B}}$-as well as of other parameters - on the power spectrum and the CMB anisotropies (see also [39, 50]). We agree with the results of Fig. 2 of this reference, for the corresponding values of $\alpha_{\mathrm{B}, 0}$ and $\alpha_{\mathrm{K}, 0} \cdot{ }^{12}$

From the above assumptions it follows that the speed of scalar fluctuations is

$$
c_{s}^{2}=-\frac{\alpha_{\mathrm{B}}\left[2\left(1+\alpha_{\mathrm{B}}\right)+3 \Omega_{\mathrm{m}}\right]}{\alpha_{\mathrm{K}}+6 \alpha_{\mathrm{B}}^{2}} .
$$

Absence of ghosts and gradient instabilities therefore imply respectively that $\alpha_{\mathrm{K}}+6 \alpha_{\mathrm{B}}^{2} \geq 0$ and $-1-(3 / 2) \Omega_{\mathrm{m}} \leq \alpha_{\mathrm{B}} \leq 0$. As in Sec. 4, we set $\alpha_{\mathrm{K}, 0}=1$; then we study the effect of $\alpha_{\mathrm{B}}$ for four negative values of $\alpha_{\mathrm{B}, 0}$ to avoid instabilities: $\alpha_{\mathrm{B}, 0}=-0.06,-0.12,-0.24$ and -0.48 .

\footnotetext{
${ }^{12}$ We can compare with Ref. [45] by the following correspondence between our parameters $\alpha_{\mathrm{B}, 0}$ and $\alpha_{\mathrm{K}, 0}$ and their parameters $\hat{\alpha}_{\mathrm{B}}$ and $\hat{\alpha}_{\mathrm{K}}: \alpha_{\mathrm{B}, 0}=-\hat{\alpha}_{\mathrm{B}} \Omega_{\mathrm{DE}, 0} / 2$ and $\alpha_{\mathrm{K}, 0}=\hat{\alpha}_{\mathrm{K}} \Omega_{\mathrm{DE}, 0}$.
} 

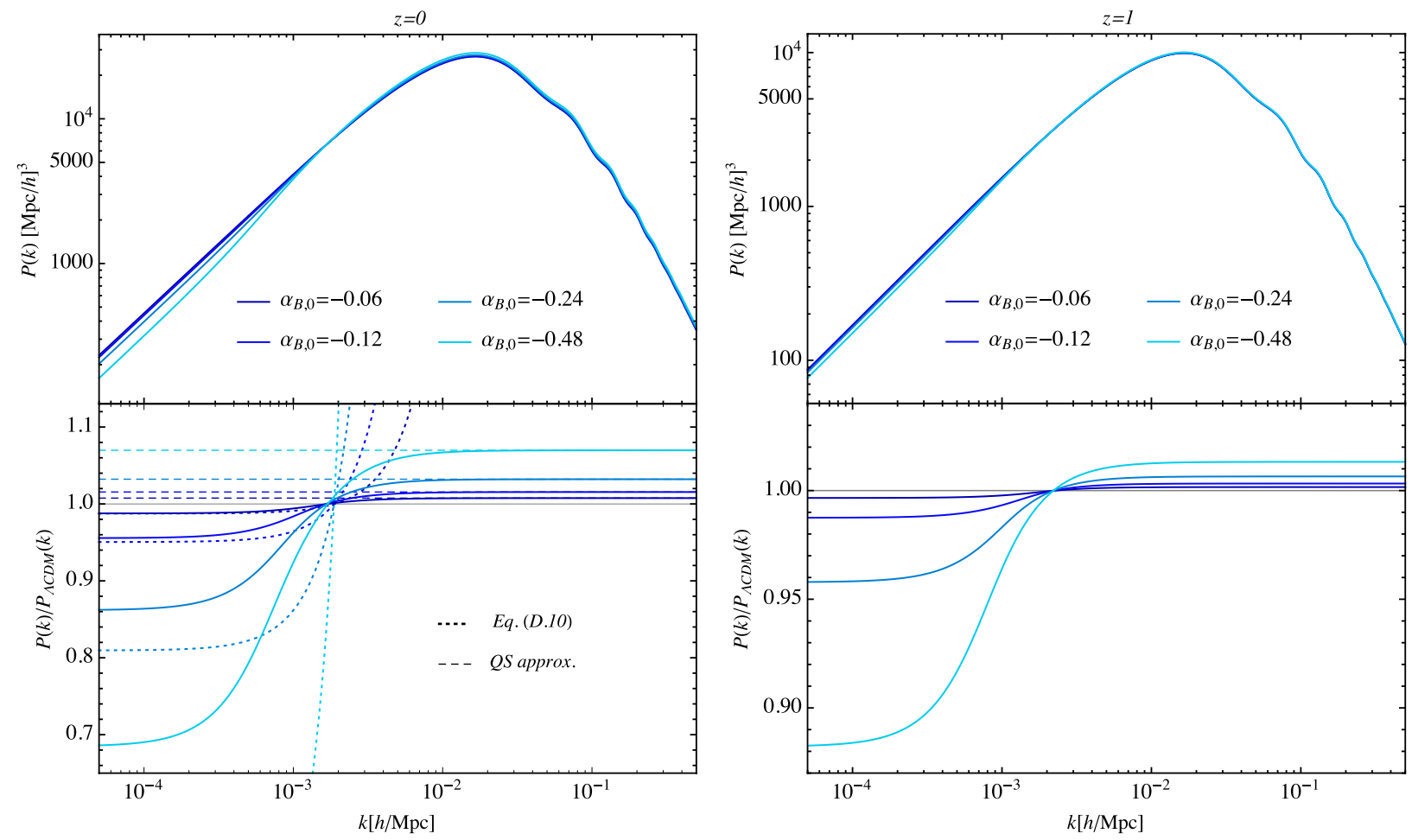

Figure 6: Matter power spectrum for four different values of $\alpha_{\mathrm{B}}$ today, i.e. $\alpha_{\mathrm{B}, 0}=-0.06,-0.12,-0.24$ and -0.48 , at redshift $z=0$ (left panel) and $z=1$ (right panel). For comparison, the dashed and dotted lines in the left lower panel respectively show the quasi-static approximation and the perturbative solution of eq. (D.10).

\section{D.1 Matter power spectrum}

We plot the matter power spectrum in Fig. 6. The effect of $\alpha_{\mathrm{B}}$ is to enhance the power on short scales, due to strengthening of gravity. Indeed, the modification of the Poisson equation (3.24) reads

$$
\mu_{\Phi}=1-\frac{\alpha_{\mathrm{B}}}{1+\alpha_{\mathrm{B}}+3 \Omega_{\mathrm{m}} / 2}
$$

and one can use this relation in eq. (3.27) (with $\gamma=0$ ) to predict the corresponding enhancement. On large scales we observe the opposite effect, i.e. a suppression of power, and a crossover scale between these two regimes independent of $\alpha_{\mathrm{B}, 0}$.

To study the large scale regime we proceed analogously to what done in Sec. 4.1 and solve the 
Einstein equations perturbatively in $\alpha_{\mathrm{B}}$. In this case, the relevant equations are

$$
\begin{aligned}
\dot{\Phi}+H \Phi-\dot{H} v_{\mathrm{m}} & =\alpha_{\mathrm{B}} H(\dot{\pi}-\Phi) \\
-\frac{k^{2}}{a^{2}} \Phi+\dot{H} \Delta_{\mathrm{m}}-\frac{\alpha_{\mathrm{K}}}{2} H^{2}(\dot{\pi}-\Phi) & =\alpha_{\mathrm{B}} H\left[3 \dot{\Phi}+3 H \Phi+3 \dot{H} \pi-\frac{k^{2}}{a^{2}} H \pi\right], \\
\ddot{\Phi}+4 H \dot{\Phi}+\left(2 \dot{H}+3 H^{2}\right) \Phi & =\alpha_{\mathrm{B}}\left[\ddot{\pi}-\dot{\Phi}+\left(3 H^{2}-\dot{H}\right)(\dot{\pi}-\Phi)\right], \\
\frac{1}{2}\left(\alpha_{\mathrm{K}}+6 \alpha_{\mathrm{B}}^{2}\right) H^{2} a^{-3} \frac{d}{d t}\left[a^{3}(\dot{\pi}-\Phi)\right]= & \alpha_{\mathrm{B}}\left\{\frac{k^{2}}{a^{2}}\left[H \Phi+\left(H^{2}-\dot{H}\right) \pi\right]\right. \\
& \left.+3 \dot{H}\left[2 \dot{\Phi}+2 H \Phi+\dot{H}\left(\pi-v_{\mathrm{m}}\right)\right]\right\} .
\end{aligned}
$$

Equation (D.5) is the " $0 i$ " scalar component of the Einstein equations, eq. (D.6) follows from combining the "00" component with eq. (D.5), eq. (D.7) is the trace of the " $i j$ " components and we have used $\Psi=\Phi$, which follows from the traceless part of the "ij" components. Finally, eq. (D.8) is the evolution equation for $\pi$.

For $\alpha_{\mathrm{B}}=0$, these equations have the solution given in eqs. (4.15) and $\pi=-\epsilon$. As in the case of $\alpha_{\mathrm{H}}, \alpha_{\mathrm{B}}$ does not affect the metric and matter perturbations at first order: eq. (4.15) remains a solution with

$$
\pi=-\epsilon-2 \frac{\alpha_{\mathrm{B}}}{\alpha_{\mathrm{K}}} k^{2} \int \frac{\epsilon d t}{a^{2} H}+\mathcal{O}\left(\alpha_{\mathrm{B}}^{2}\right) .
$$

In order to see the effects of braiding we need to go at second order in $\alpha_{\mathrm{B}}$ [5]. The matter density contrast $\Delta_{\mathrm{m}}$ can be computed from eq. (D.6), similarly to what discussed in Sec. 4.1. We can solve for $\Phi$ at second order from eq. (D.7), where we use the first-order solution on the righthand side. To derive $\dot{\pi}-\Phi$, we can solve eq. (D.8) after replacing $v_{\mathrm{m}}$ using (D.6). In conclusion, the density constrast reads

$$
\Delta_{\mathrm{m}}=\Delta_{\mathrm{m}, \Lambda \mathrm{CDM}}\left[1-2 \frac{\alpha_{\mathrm{B}}^{2}}{\alpha_{\mathrm{K}}}\left(F_{1}-\frac{k^{2}}{a^{2} H^{2}} F_{2}\right)\left(1-\frac{H}{a} \int a d t\right)^{-1}+\mathcal{O}\left(\alpha_{\mathrm{B}}^{4}\right)\right],
$$

where

$$
\begin{aligned}
& F_{1} \equiv \frac{3 H^{2}}{a}\left(\frac{1}{H} \int a d t-\int \frac{a d t}{H}\right) \\
& F_{2} \equiv a H^{4}\left[H \int \frac{1}{a^{2} H^{2}}\left(\frac{2}{H} \int a d t-\int \frac{a d t}{H}\right)-\int \frac{d t}{a^{2} H^{2}} \int a d t\right] .
\end{aligned}
$$

Equation (D.10) explains the large scale suppression in the power spectrum and why the crossover scale, which can be derive from the above equation as

$$
k_{\mathrm{c}}=a H \sqrt{F_{1} / F_{2}},
$$

is independent of $\alpha_{\mathrm{B}}$. However, in Fig. (6) we observe a large discrepancy between eq. (D.10) and the output of COOP. It can be checked that the difference grows as $\alpha_{\mathrm{B}}^{4}$ and it is thus due to the neglected corrections to eq. (D.10). 

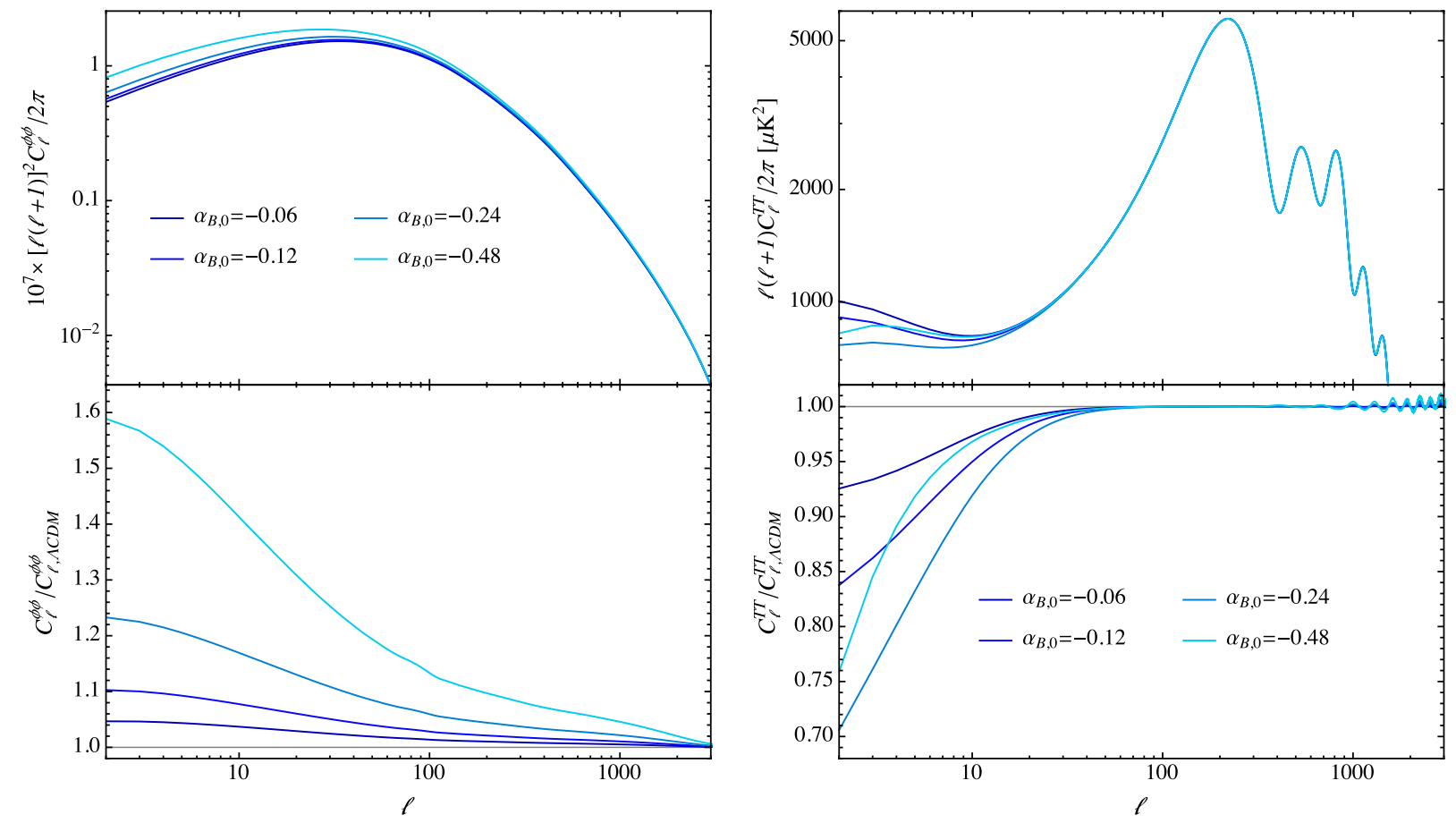

Figure 7: Effect of braiding $\left(\alpha_{\mathrm{B}}\right)$ on the CMB lensing potential (left panel) and on the CMB anisotropies (right panel) angular power spectra. The lower plots display the ratio of these angular spectra with the respective spectra for $\alpha_{\mathrm{B}}=0$.

\section{D.2 Cosmic microwave background}

In Fig. 7 we plot the angular power spectrum of the lensing potential (left panel) and of the CMB anisotropies (right panel). A negative braiding parameter $\alpha_{\mathrm{B}}$ induces an enhancement in the lensing potential. Similarly to what done in the previous section, we can understand this effect as a modification of the Weyl potential, expressed in terms of the parameter $\mu_{\mathrm{WL}}$ in eq. (4.23). Setting $\alpha_{\mathrm{M}}=\alpha_{\mathrm{T}}=\alpha_{\mathrm{H}}=0$, this reads (see also [35] for an analysis using the quasi-static approximation)

$$
\mu_{\mathrm{WL}}-2=-\frac{2 \alpha_{\mathrm{B}}}{1+\alpha_{\mathrm{B}}+3 \Omega_{\mathrm{m}} / 2} .
$$

This relation shows that for negative values of $\alpha_{\mathrm{B}}$, the Weyl potential is enhanced for all redshifts. Comparing with the effect of $\alpha_{\mathrm{H}}$ shown in Fig. 3, we notice that here the effect is larger at smaller $l$; this is due to the fact that, contrarily to the $\alpha_{\mathrm{H}}$ case, here $\mu_{\mathrm{WL}}-2$ does not change sign at low redshift, and contributes also to low multipoles.

Let us turn now to the CMB angular power spectrum, right panel of Fig. 7. Increasing $-\alpha_{\mathrm{B}}$ enhances the lensing potential, thus increasing the smearing effect on the CMB acoustic peaks, as shown on the right lower panel. The suppression of the ISW effect can be understood again by looking at eq. (4.26). Now

$$
\frac{d \ln \mu_{\mathrm{WL}}}{d \ln a}=-\frac{15 \alpha_{\mathrm{B}} \Omega_{\mathrm{m}}}{\left(2+3 \Omega_{\mathrm{m}}\right)\left(1+\alpha_{\mathrm{B}}+3 \Omega_{\mathrm{m}} / 2\right)},
$$


which for negative values of $\alpha_{\mathrm{B}}$ is positive, i.e. has opposite sign as the standard $\Lambda$ CDM contribution coming from the first two terms in the right-hand side of eq. (4.26). For small values of $-\alpha_{\mathrm{B}, 0}$, $d \ln \mu_{\mathrm{WL}} / d \ln a$ is smaller than $1-d \ln \delta_{\mathrm{m}} / d \ln a$ : the time derivative of the Weyl potential remains negative and the net ISW effect is suppressed by kinetic braiding. For large values of $-\alpha_{\mathrm{B}, 0}$, i.e. $-\alpha_{\mathrm{B}, 0} \gtrsim 0.3$, the right-hand side of eq. (4.26) changes sign and increasing $\alpha_{\mathrm{B}}$ enhances the ISW effect.

\section{References}

[1] Euclid Theory Working Group Collaboration, L. Amendola et. al., "Cosmology and fundamental physics with the Euclid satellite," Living Rev. Rel. 16 (2013) 6, 1206.1225.

[2] L. Amendola et. al., "Cosmology and Fundamental Physics with the Euclid Satellite," 1606.00180.

[3] T. Clifton, P. G. Ferreira, A. Padilla, and C. Skordis, "Modified Gravity and Cosmology," Phys.Rept. 513 (2012) 1-189, 1106.2476.

[4] A. Joyce, B. Jain, J. Khoury, and M. Trodden, "Beyond the Cosmological Standard Model," Phys.Rept. 568 (2015) 1-98, 1407.0059.

[5] P. Creminelli, G. D’Amico, J. Norena, and F. Vernizzi, "The Effective Theory of Quintessence: the $w<-1$ Side Unveiled," JCAP 0902 (2009) 018, 0811.0827.

[6] G. Gubitosi, F. Piazza, and F. Vernizzi, "The Effective Field Theory of Dark Energy," JCAP 1302 (2013) 032, 1210.0201.

[7] J. K. Bloomfield, É. É. Flanagan, M. Park, and S. Watson, "Dark energy or modified gravity? An effective field theory approach," JCAP 1308 (2013) 010, 1211.7054.

[8] J. Gleyzes, D. Langlois, F. Piazza, and F. Vernizzi, "Essential Building Blocks of Dark Energy," JCAP 1308 (2013) 025, 1304.4840.

[9] J. Bloomfield, "A Simplified Approach to General Scalar-Tensor Theories," JCAP 1312 (2013) 044, 1304.6712.

[10] J. Gleyzes, D. Langlois, and F. Vernizzi, "A unifying description of dark energy," Int. J. Mod. Phys. D23 (2015), no. 13 1443010, 1411.3712.

[11] R. A. Battye and J. A. Pearson, "Effective action approach to cosmological perturbations in dark energy and modified gravity," JCAP 1207 (2012) 019, 1203.0398.

[12] R. A. Battye and J. A. Pearson, "Computing model independent perturbations in dark energy and modified gravity," JCAP 1403 (2014) 051, 1311.6737.

[13] T. Baker, P. G. Ferreira, C. Skordis, and J. Zuntz, "Towards a fully consistent parameterization of modified gravity," Phys. Rev. D84 (2011) 124018, 1107.0491.

[14] T. Baker, P. G. Ferreira, and C. Skordis, "The Parameterized Post-Friedmann framework for theories of modified gravity: concepts, formalism and examples," Phys. Rev. D87 (2013), no. 2 024015, 1209.2117. 
[15] C. Skordis, A. Pourtsidou, and E. J. Copeland, "Parametrized post-Friedmannian framework for interacting dark energy theories," Phys. Rev. D91 (2015), no. 8083537 , 1502.07297.

[16] M. Lagos, T. Baker, P. G. Ferreira, and J. Noller, "A general theory of linear cosmological perturbations: scalar-tensor and vector-tensor theories," JCAP 1608 (2016), no. 08 007, 1604.01396.

[17] E. Bellini, R. Jimenez, and L. Verde, "Signatures of Horndeski gravity on the Dark Matter Bispectrum," JCAP 1505 (2015), no. 05 057, 1504.04341.

[18] E. Bellini and M. Zumalacarregui, "Nonlinear evolution of the baryon acoustic oscillation scale in alternative theories of gravity," Phys. Rev. D92 (2015), no. 6 063522, 1505.03839.

[19] P. Creminelli, M. A. Luty, A. Nicolis, and L. Senatore, "Starting the Universe: Stable Violation of the Null Energy Condition and Non-standard Cosmologies," JHEP 0612 (2006) 080, hep-th/0606090.

[20] C. Cheung, P. Creminelli, A. L. Fitzpatrick, J. Kaplan, and L. Senatore, "The Effective Field Theory of Inflation," JHEP 0803 (2008) 014, 0709.0293.

[21] F. Piazza and F. Vernizzi, "Effective Field Theory of Cosmological Perturbations," Class.Quant.Grav. 30 (2013) 214007, 1307.4350.

[22] S. Tsujikawa, "The effective field theory of inflation/dark energy and the Horndeski theory," Lect.Notes Phys. 892 (2015) 97-136, 1404.2684.

[23] G. W. Horndeski, "Second-order scalar-tensor field equations in a four-dimensional space," Int.J.Theor.Phys. 10 (1974) 363-384.

[24] C. Deffayet, X. Gao, D. Steer, and G. Zahariade, "From k-essence to generalised Galileons," Phys.Rev. D84 (2011) 064039, 1103.3260.

[25] T. Kobayashi, M. Yamaguchi, and J. Yokoyama, "Generalized G-inflation: Inflation with the most general second-order field equations," Prog.Theor.Phys. 126 (2011) 511-529, 1105.5723 .

[26] E. Bellini and I. Sawicki, "Maximal freedom at minimum cost: linear large-scale structure in general modifications of gravity," JCAP 1407 (2014) 050, 1404.3713.

[27] J. Gleyzes, D. Langlois, F. Piazza, and F. Vernizzi, "Healthy theories beyond Horndeski," Phys. Rev. Lett. 114 (2015), no. 21 211101, 1404.6495.

[28] J. Gleyzes, D. Langlois, F. Piazza, and F. Vernizzi, "Exploring gravitational theories beyond Horndeski," JCAP 1502 (2015) 018, 1408.1952.

[29] M. Zumalacárregui and J. García-Bellido, "Transforming gravity: from derivative couplings to matter to second-order scalar-tensor theories beyond the Horndeski Lagrangian," Phys.Rev. D89 (2014), no. 6 064046, 1308.4685.

[30] F. Piazza, H. Steigerwald, and C. Marinoni, "Phenomenology of dark energy: exploring the space of theories with future redshift surveys," JCAP 1405 (2014) 043, 1312.6111. 
[31] R. Kase and S. Tsujikawa, "Cosmology in generalized Horndeski theories with second-order equations of motion," Phys. Rev. D90 (2014) 044073, 1407.0794.

[32] Planck Collaboration, P. Ade et. al., "Planck 2015 results. XIV. Dark energy and modified gravity," 1502.01590.

[33] L. Lombriser and A. Taylor, "Semi-dynamical perturbations of unified dark energy," JCAP 1511 (2015), no. 11 040, 1505.05915.

[34] L. Perenon, F. Piazza, C. Marinoni, and L. Hui, "Phenomenology of dark energy: general features of large-scale perturbations," JCAP 1511 (2015), no. 11 029, 1506.03047.

[35] J. Gleyzes, D. Langlois, M. Mancarella, and F. Vernizzi, "Effective Theory of Dark Energy at Redshift Survey Scales," JCAP 1602 (2016), no. 02 056, 1509.02191.

[36] N. Frusciante, G. Papadomanolakis, and A. Silvestri, "An Extended action for the effective field theory of dark energy: a stability analysis and a complete guide to the mapping at the basis of EFTCAMB," JCAP 1607 (2016), no. 07 018, 1601.04064.

[37] B. Hu, M. Raveri, M. Rizzato, and A. Silvestri, "Testing Hu?Sawicki f(R) gravity with the effective field theory approach," Mon. Not. Roy. Astron. Soc. 459 (2016), no. 4 3880-3889, 1601.07536.

[38] V. Salvatelli, F. Piazza, and C. Marinoni, "Constraints on modified gravity from Planck 2015: when the health of your theory makes the difference," 1602.08283.

[39] J. Renk, M. Zumalacarregui, and F. Montanari, "Gravity at the horizon: on relativistic effects, CMB-LSS correlations and ultra-large scales in Horndeski's theory," JCAP 1607 (2016), no. 07 040, 1604.03487.

[40] J. S. Y. Leung and Z. Huang, "Marginalized Fisher Forecast for Horndeski Dark Energy Models," 1604.07330.

[41] L. Pogosian and A. Silvestri, "What can Cosmology tell us about Gravity? Constraining Horndeski with Sigma and Mu," 1606.05339.

[42] B. Hu, M. Raveri, N. Frusciante, and A. Silvestri, "Effective Field Theory of Cosmic Acceleration: an implementation in CAMB," Phys.Rev. D89 (2014), no. 10103530 , 1312.5742.

[43] M. Raveri, B. Hu, N. Frusciante, and A. Silvestri, "Effective Field Theory of Cosmic Acceleration: constraining dark energy with CMB data," Phys. Rev. D90 (2014), no. 4 043513, 1405.1022.

[44] E. Bellini, A. J. Cuesta, R. Jimenez, and L. Verde, "Constraints on deviations from CDM within Horndeski gravity," JCAP 1602 (2016), no. 02 053, 1509.07816. [Erratum: JCAP1606,no.06,E01(2016)].

[45] M. Zumalacárregui, E. Bellini, I. Sawicki, and J. Lesgourgues, "hi_class: Horndeski in the Cosmic Linear Anisotropy Solving System," 1605.06102. 
[46] Z. Huang, "Observational effects of a running Planck mass," Phys. Rev. D93 (2016), no. 4 043538, 1511.02808.

[47] Z. Huang, "COOP: first release; EFTDE/XFASTER/CPLDE," August, 2016. http://dx.doi.org/10.5281/zenodo.61166.

[48] C. M. Will, "The Confrontation between General Relativity and Experiment," Living Rev.Rel. 17 (2014) 4, 1403.7377.

[49] G. Domènech, S. Mukohyama, R. Namba, A. Naruko, R. Saitou, and Y. Watanabe, "Derivative-dependent metric transformation and physical degrees of freedom," Phys. Rev. D92 (2015), no. 8 084027, 1507.05390.

[50] J. Gleyzes, D. Langlois, M. Mancarella, and F. Vernizzi, "Effective Theory of Interacting Dark Energy," JCAP 1508 (2015), no. 08 054, 1504.05481.

[51] D. Bettoni and S. Liberati, "Disformal invariance of second order scalar-tensor theories: Framing the Horndeski action," Phys.Rev. D88 (2013), no. 8 084020, 1306.6724.

[52] I. Sawicki and E. Bellini, "Limits of quasistatic approximation in modified-gravity cosmologies," Phys. Rev. D92 (2015), no. 8 084061, 1503.06831.

[53] D. Bettoni and M. Zumalacárregui, "Kinetic mixing in scalar-tensor theories of gravity," Phys. Rev. D91 (2015) 104009, 1502.02666.

[54] M. Crisostomi, M. Hull, K. Koyama, and G. Tasinato, "Horndeski: beyond, or not beyond?," JCAP 1603 (2016), no. 03 038, 1601.04658.

[55] D. Langlois and K. Noui, "Degenerate higher derivative theories beyond Horndeski: evading the Ostrogradski instability," JCAP 1602 (2016), no. 02 034, 1510.06930.

[56] J. Ben Achour, D. Langlois, and K. Noui, "Degenerate higher order scalar-tensor theories beyond Horndeski and disformal transformations," Phys. Rev. D93 (2016), no. 12 124005, 1602.08398.

[57] M. Crisostomi, K. Koyama, and G. Tasinato, "Extended Scalar-Tensor Theories of Gravity," JCAP 1604 (2016), no. 04 044, 1602.03119.

[58] T. Kobayashi, Y. Watanabe, and D. Yamauchi, "Breaking of Vainshtein screening in scalar-tensor theories beyond Horndeski," Phys. Rev. D91 (2015), no. 6 064013, 1411.4130.

[59] A. De Felice, K. Koyama, and S. Tsujikawa, "Observational signatures of the theories beyond Horndeski," JCAP 1505 (2015), no. 05 058, 1503.06539.

[60] R. Saito, D. Yamauchi, S. Mizuno, J. Gleyzes, and D. Langlois, "Modified gravity inside astrophysical bodies," JCAP 1506 (2015) 008, 1503.01448.

[61] R. Kase, S. Tsujikawa, and A. De Felice, "Cosmology with a successful Vainshtein screening in theories beyond Horndeski," Phys. Rev. D93 (2016), no. 2 024007, 1510.06853. 
[62] J. Sakstein, H. Wilcox, D. Bacon, K. Koyama, and R. C. Nichol, "Testing Gravity Using Galaxy Clusters: New Constraints on Beyond Horndeski Theories," JCAP 1607 (2016), no. 07 019, 1603.06368.

[63] E. Babichev, K. Koyama, D. Langlois, R. Saito, and J. Sakstein, "Relativistic Stars in Beyond Horndeski Theories," 1606.06627.

[64] C. Deffayet, O. Pujolas, I. Sawicki, and A. Vikman, "Imperfect Dark Energy from Kinetic Gravity Braiding," JCAP 1010 (2010) 026, 1008.0048.

[65] O. Pujolas, I. Sawicki, and A. Vikman, "The Imperfect Fluid behind Kinetic Gravity Braiding," JHEP 11 (2011) 156, 1103.5360.

[66] Planck Collaboration Collaboration, P. Ade et. al., "Planck 2013 results. XVI. Cosmological parameters," Astron.Astrophys. 571 (2014) A16, 1303.5076.

[67] Planck Collaboration, P. A. R. Ade et. al., "Planck 2015 results. XIII. Cosmological parameters," 1502.01589.

[68] L. Á. Gergely and S. Tsujikawa, "Effective field theory of modified gravity with two scalar fields: dark energy and dark matter," Phys.Rev. D89 (2014), no. 6 064059, 1402.0553.

[69] G. D. Moore and A. E. Nelson, "Lower bound on the propagation speed of gravity from gravitational Cherenkov radiation," JHEP 0109 (2001) 023, hep-ph/0106220.

[70] J. Beltran Jimenez, F. Piazza, and H. Velten, "Evading the Vainshtein Mechanism with Anomalous Gravitational Wave Speed: Constraints on Modified Gravity from Binary Pulsars," Phys. Rev. Lett. 116 (2016), no. 6 061101, 1507.05047.

[71] Z. Huang, “A Cosmology Forecast Toolkit - CosmoLib," JCAP 1206 (2012) 012, 1201.5961.

[72] U. Seljak and M. Zaldarriaga, "A Line of sight integration approach to cosmic microwave background anisotropies," Astrophys. J. 469 (1996) 437-444, astro-ph/9603033.

[73] W. Hu and M. J. White, "CMB anisotropies: Total angular momentum method," Phys. Rev. D56 (1997) 596-615, astro-ph/9702170.

[74] A. Iglesias, N. Kaloper, A. Padilla, and M. Park, "How (Not) to Palatini," Phys. Rev. D76 (2007) 104001, 0708.1163.

[75] N. Arkani-Hamed, H.-C. Cheng, M. A. Luty, and S. Mukohyama, "Ghost condensation and a consistent infrared modification of gravity," JHEP 05 (2004) 074, hep-th/0312099.

[76] A. Lewis and A. Challinor, "Weak gravitational lensing of the cmb," Phys. Rept. 429 (2006) 1-65, astro-ph/0601594.

[77] M. Kilbinger et. al., "CFHTLenS: Combined probe cosmological model comparison using 2D weak gravitational lensing," Mon. Not. Roy. Astron. Soc. 430 (2013) 2200-2220, 1212.3338. 
[78] C. Heymans et. al., "CFHTLenS tomographic weak lensing cosmological parameter constraints: Mitigating the impact of intrinsic galaxy alignments," Mon. Not. Roy. Astron. Soc. 432 (2013) 2433, 1303.1808.

[79] CFHTLenS Collaboration, T. D. Kitching et. al., "3D Cosmic Shear: Cosmology from CFHTLenS," Mon. Not. Roy. Astron. Soc. 442 (2014), no. 2 1326-1349, 1401.6842.

[80] F. Köhlinger, M. Viola, W. Valkenburg, B. Joachimi, H. Hoekstra, and K. Kuijken, "A direct measurement of tomographic lensing power spectra from CFHTLenS," Mon. Not. Roy. Astron. Soc. 456 (2016), no. 2 1508-1527, 1509.04071.

[81] Planck Collaboration, P. A. R. Ade et. al., "Planck 2013 results. XX. Cosmology from Sunyaev-Zeldovich cluster counts," Astron. Astrophys. 571 (2014) A20, 1303.5080.

[82] Planck Collaboration, P. A. R. Ade et. al., "Planck 2015 results. XXIV. Cosmology from Sunyaev-Zeldovich cluster counts," 1502.01597.

[83] SPT Collaboration, T. de Haan et. al., "Cosmological Constraints from Galaxy Clusters in the 2500 square-degree SPT-SZ Survey," Submitted to: Astrophys. J. (2016) 1603.06522.

[84] H. Hildebrandt et. al., "KiDS-450: Cosmological parameter constraints from tomographic weak gravitational lensing," 1606.05338.

[85] BOSS Collaboration, S. Alam et. al., "The clustering of galaxies in the completed SDSS-III Baryon Oscillation Spectroscopic Survey: cosmological analysis of the DR12 galaxy sample," Submitted to: Mon. Not. Roy. Astron. Soc. (2016) 1607.03155.

[86] E. Macaulay, I. K. Wehus, and H. K. Eriksen, "Lower Growth Rate from Recent Redshift Space Distortion Measurements than Expected from Planck," Phys. Rev. Lett. 111 (2013), no. 16 161301, 1303.6583.

[87] DES Collaboration, T. Abbott et. al., "Cosmology from cosmic shear with Dark Energy Survey Science Verification data," Phys. Rev. D94 (2016), no. 2 022001, 1507.05552.

[88] S. Joudaki et. al., "CFHTLenS revisited: assessing concordance with Planck including astrophysical systematics," 1601.05786.

[89] M. Wyman, D. H. Rudd, R. A. Vanderveld, and W. Hu, "Neutrinos Help Reconcile Planck Measurements with the Local Universe," Phys. Rev. Lett. 112 (2014), no. 5 051302, 1307.7715 .

[90] R. A. Battye, T. Charnock, and A. Moss, "Tension between the power spectrum of density perturbations measured on large and small scales," Phys. Rev. D91 (2015), no. 10103508 , 1409.2769.

[91] M. Kunz, S. Nesseris, and I. Sawicki, "Using dark energy to suppress power at small scales," Phys. Rev. D92 (2015), no. 6 063006, 1507.01486.

[92] M. Kunz, "The dark degeneracy: On the number and nature of dark components," Phys. Rev. D80 (2009) 123001, astro-ph/0702615. 
[93] P. Creminelli, G. D’Amico, J. Norena, L. Senatore, and F. Vernizzi, "Spherical collapse in quintessence models with zero speed of sound," JCAP 1003 (2010) 027, 0911.2701.

[94] E. Sefusatti and F. Vernizzi, "Cosmological structure formation with clustering quintessence," JCAP 1103 (2011) 047, 1101.1026.

[95] S. Tsujikawa, "Possibility of realizing weak gravity in redshift space distortion measurements," Phys. Rev. D92 (2015), no. 4 044029, 1505.02459.

[96] F. Beutler, C. Blake, M. Colless, D. H. Jones, L. Staveley-Smith, G. B. Poole, L. Campbell, Q. Parker, W. Saunders, and F. Watson, "The 6dF Galaxy Survey: $z \approx 0$ measurement of the growth rate and $\sigma_{8}, "$ Mon. Not. Roy. Astron. Soc. 423 (2012) 3430-3444, 1204.4725.

[97] C. Howlett, A. Ross, L. Samushia, W. Percival, and M. Manera, "The clustering of the SDSS main galaxy sample? II. Mock galaxy catalogues and a measurement of the growth of structure from redshift space distortions at $z=0.15$," Mon. Not. Roy. Astron. Soc. 449 (2015), no. 1 848-866, 1409.3238.

[98] C. Blake et. al., "Galaxy And Mass Assembly (GAMA): improved cosmic growth measurements using multiple tracers of large-scale structure," Mon. Not. Roy. Astron. Soc. 436 (2013) 3089, 1309.5556.

[99] C. Blake et. al., "The WiggleZ Dark Energy Survey: Joint measurements of the expansion and growth history at $z<1, "$ Mon. Not. Roy. Astron. Soc. 425 (2012) 405-414, 1204.3674.

[100] S. de la Torre et. al., "The VIMOS Public Extragalactic Redshift Survey (VIPERS). Galaxy clustering and redshift-space distortions at $\mathrm{z}=0.8$ in the first data release," Astron. Astrophys. 557 (2013) A54, 1303.2622.

[101] C. Armendariz-Picon, V. F. Mukhanov, and P. J. Steinhardt, "A Dynamical solution to the problem of a small cosmological constant and late time cosmic acceleration," Phys.Rev.Lett. 85 (2000) 4438-4441, astro-ph/0004134.

[102] C. Armendariz-Picon, V. F. Mukhanov, and P. J. Steinhardt, "Essentials of k essence," Phys.Rev. D63 (2001) 103510, astro-ph/0006373.

[103] L. Boubekeur, P. Creminelli, J. Norena, and F. Vernizzi, "Action approach to cosmological perturbations: the 2nd order metric in matter dominance," JCAP 0808 (2008) 028, 0806.1016.

[104] J. M. Maldacena, "Non-Gaussian features of primordial fluctuations in single field inflationary models," JHEP 0305 (2003) 013, astro-ph/0210603. 